\title{
The Isaac Newton Telescope Monitoring Survey of Local Group Dwarf Galaxies. IV. The Star Formation History of Andromeda VII Derived from Long-period Variable Stars
}

\author{
Mahdieh Navabi ${ }^{1}$, Elham Saremi ${ }^{1}$, Atefeh Javadi ${ }^{1,2}$, Majedeh Noori ${ }^{3}$, Jacco Th. van Loon ${ }^{4}$ (1), Habib G. Khosroshahi ${ }^{1,5}$, \\ Iain McDonald ${ }^{6,7}$, Mina Alizadeh ${ }^{1,3}$, Arash Danesh ${ }^{5}$, Ghassem Gozaliasl ${ }^{8,9,10}$, Alireza Molaeinezhad ${ }^{11,12}$, Tahere Parto ${ }^{1,13}$, and \\ Mojtaba Raouf ${ }^{14}$ (1) \\ ${ }^{1}$ School of Astronomy, Institute for Research in Fundamental Sciences (IPM), P.O. Box 1956836613, Tehran, Iran; mahdieh.navabi@ipm.ir \\ ${ }_{2}^{2}$ Department of Physics, Sharif University of Technology, P.O. Box 11155-9161, Tehran, Iran \\ ${ }^{3}$ Department of Physics, University of Zanjan, University Blvd., P.O. Box 45371-38791, Zanjan, Iran \\ ${ }^{4}$ Lennard-Jones Laboratories, Keele University, ST5 5BG, UK \\ ${ }^{5}$ Iranian National Observatory, Institute for Research in Fundamental Sciences (IPM), Tehran, Iran \\ ${ }^{6}$ Jodrell Bank Centre for Astrophysics, University of Manchester, M13 9PL, UK \\ ${ }^{7}$ Department of Physical Sciences, The Open University, Walton Hall, Milton Keynes, MK7 6AA, UK \\ ${ }^{8}$ Finnish Centre for Astronomy with ESO (FINCA), Quantum, University of Turku, Vesilinnantie 5, FI-20014 Turku, Finland \\ ${ }^{9}$ Department of Physics, University of Helsinki, P.O. Box 64, FI-00014 Helsinki, Finland \\ ${ }^{10}$ Helsinki Institute of Physics, University of Helsinki, P.O. Box 64, FI-00014 Helsinki, Finland \\ ${ }_{11}$ Department of Physics, University of Oxford, Keble Road, OX1 3RH Oxford, UK \\ ${ }^{12}$ Institute of Astronomy, University of Cambridge, Madingley Road, Cambridge CB3 OHA, UK \\ ${ }^{13}$ Physics Department, Alzahra University, Vanak, 1993891176, Tehran, Iran \\ ${ }^{14}$ Korea Astronomy and Space Science Institute, 776 Daedeokdae-ro, Yuseong-gu, Daejeon 34055, Republic of Korea \\ Received 2020 November 12; revised 2021 January 16; accepted 2021 January 18; published 2021 April 5
}

\begin{abstract}
We have examined the star formation history (SFH) of Andromeda VII (And VII), the brightest and most massive dwarf spheroidal (dSph) satellite of the Andromeda galaxy (M31). Although M31 is surrounded by several $\mathrm{dSph}$ companions with old stellar populations and low metallicity, it has a metal-rich stellar halo with an age of 6-8 Gyr. This indicates that any evolutionary association between the stellar halo of M31 and its dSph system is frail. Therefore, the question is whether And VII (a high-metallicity dSph located 220 kpc from M31) can be associated with M31's young, metal-rich halo. Here we perform the first reconstruction of the SFH of And VII employing long-period variable (LPV) stars. As the most evolved asymptotic giant branch and red supergiant stars, the birth mass of LPVs can be determined by connecting their near-infrared photometry to theoretical evolutionary tracks. We found 55 LPV candidates within two half-light radii, using multiepoch imaging with the Isaac Newton Telescope in the $i$ and $V$ bands. Based on their birth mass function, the star formation rate (SFR) of And VII was obtained as a function of cosmic time. The main epoch of star formation occurred $\simeq 6.2$ Gyr ago with an SFR of $0.006 \pm 0.002 M_{\odot} \mathrm{yr}^{-1}$. Over the past $6 \mathrm{Gyr}$, we find slow star formation, which continued until $500 \mathrm{Myr}$ ago with an SFR $\sim 0.0005 \pm 0.0002 M_{\odot} \mathrm{yr}^{-1}$. We determined And VII's stellar mass $M=(13.3 \pm 5.3) \times 10^{6} M_{\odot}$ within a half-light radius $r_{\frac{1}{2}}=3.8 \pm 0.3$ and metallicity $Z=0.0007$, and we also derived its distance modulus of $\mu=24.38$ mag.
\end{abstract}

Unified Astronomy Thesaurus concepts: Dwarf spheroidal galaxies (420); Star formation (1569)

\section{Introduction}

Dwarf spheroidal (dSph) galaxies are early-type galaxies devoid of gas and dust. As most dSphs lie in the halos of their massive host galaxies, their gas has been stripped through interactions with their host galaxies, and thus their star formation has halted long ago (Battinelli \& Demers 2006; Grcevich \& Putman 2009; Tollerud et al. 2012). Since there is no sign of gaseous content and young stars in dSphs, their earlier star formation history (SFH) plays a decisive role in the scenario of formation and evolution of these low-mass galaxies. The time and speed of quenching for a dwarf can be determined from its SFH and used to quantify the effects of halo mass, infall time, and vicinity to a host, as well as stellar feedback and heating of gas at the epoch of reionization.

To explore the link between host galaxies and their dSph companions, we here study Andromeda VII, the most massive and extended dSph associated with M31. And VII (also known as the Cassiopeia dwarf) was discovered by Karachentsev and Karachentseva in Karachentsev et al. (1999) as a satellite of M31, along with two companions, And V and And VI. This satellite is located near the Galactic plane $\left(l=109^{\circ} .5, b=-10^{\circ}\right)$ and exhibits foreground reddening of $E(B-V)=0.194$ mag (McConnachie \& Irwin 2006). The distance to And VII has been determined from the tip of the red giant branch (RGB) as $\mu=24.41 \mathrm{mag}$ (763 kpc) (McConnachie et al. 2005). From scaling the color-magnitude diagram (CMD), a distance modulus of $\mu=24.5$ mag was estimated by Karachentsev et al. (2003), and $\mu=24.58$ mag by Weisz et al. (2014a).

And VII contains the largest stellar mass between all M31 dSph satellites $\left(M_{*}=19.73 \times 10^{6} M_{\odot}\right)$, and it is the eighth most massive galaxy in the entire M31-M33 group (McConnachie et al. 2018). Accordingly, it is one of the most luminous satellites of M31, with $M_{V}=-13.3 \pm 0.3 \mathrm{mag}$ and $L_{V}=1.8 \times 10^{7} L_{\odot}$ (Kalirai et al. 2010). Besides, it has a higher metallicity than most of M31's dSphs $([\mathrm{Fe} / \mathrm{H}]=-1.4 \pm 0.3$ obtained by Grebel \& Guhathakurta 1999 from a Keck/LRIS photometric study and $[\mathrm{Fe} / \mathrm{H}] \sim-1.3 \pm 0.1$ by Vargas et al. 2014; Kirby et al. 2020; Wojno et al. 2020 from spectra).

Despite the present large separation of And VII from M31 $(\sim 220 \mathrm{kpc})$, this dwarf is one of the most $\alpha$-element-enhanced systems, showing a flat trend in $[\alpha / \mathrm{Fe}]$ with $[\mathrm{Fe} / \mathrm{H}]$. This 
would suggest a closer proximity of And VII to M31 in the past, which purportedly caused it to cease star formation (Vargas et al. 2014). Furthermore, the [ $\alpha / \mathrm{Fe}]$ pattern of M31's outer halo is consistent with that of And VII (Gilbert et al. 2020). The other feature that supports an ancient, close encounter is the equal radial velocity of And VII and the peak of M31's halo velocity ( $v_{\text {rad }}=-309.4 \pm 2.3 \mathrm{~km} \mathrm{~s}^{-1}$; Tollerud et al. 2012).

To probe the star formation and enrichment histories in dwarf galaxies, CMD synthesis has been the method of choice in recent times (e.g., Dolphin 1997; Cole et al. 2007; Weisz et al. 2014a; Skillman et al. 2017; Dell'Agli et al. 2018). Despite the popularity of the CMD method, this method cannot be a suitable approach for all galaxies. For example, Weisz et al. (2014a) derived the SFH of 40 Local Group dwarf galaxies based on CMD analysis, but they admitted that they were not successful in determining the anomalous SFH of And VII and Cetus dwarfs, because their photometry was too shallow, and key CMD features used to derive the SFH were missing (e.g., horizontal branch or red clump). In this study, we employ an alternative method based on long-period variable (LPV) stars to reconstruct the SFH of And VII. This SFH method was described by Javadi et al. (2011b) and has been successfully applied to M33 and several dwarfs (Rezaeikh et al. 2014; Hamedani Golshan et al. 2017; Javadi et al. 2017; Hashemi et al. 2019; Saremi et al. 2019b).

LPV stars are mostly asymptotic giant branch (AGB) stars that reach the highest luminosity at their final stages of evolution, and hence there is a direct relationship between their luminosity and birth mass. Therefore, they enable us to derive the SFH across a broad range of ages, from $\sim 30 \mathrm{Myr}$ to $\sim 10$ Gyr (Marigo et al. 2008). In addition, to trace the more recent SFH $\sim 10-30 \mathrm{Myr}$ ago, red supergiant (RSG) stars with higher mass $\left(\sim 8-30 M_{\odot}\right)$ can be used in a similar manner (Levesque et al. 2005; Levesque 2010). The cool evolved AGB stars $\left(\sim 0.8-8 M_{\odot}\right)$ reach high luminosity $\left(\approx 1000-60,000 L_{\odot}\right)$ and low temperature $(T \approx 3000 \mathrm{~K})$ toward the end of their lifetime (Höfner \& Olofsson 2018; Yuan et al. 2018). The corresponding low surface gravity leads to strong radial pulsations, which, in turn, facilitates the production of dust and a strong stellar wind, replenishing the interstellar medium (ISM; Bladh et al. 2019). LPV stars are easily detected and identified in nearby galaxies owing to their pulsation on timescales of months to years (Saremi et al. 2019a). At the peak of their spectral energy distribution (SED), they stand out most conspicuously at near-infrared (near-IR) wavelengths, which helps diminish the effect of dust extinction and reddening (Iben \& Renzini 1983; Draine \& Li 2007).

We conducted an optical survey of nearby galaxies (the most complete sample so far) with the $2.5 \mathrm{~m}$ Isaac Newton Telescope (INT) over nine epochs (Saremi et al. 2020). Our main objectives include the following: identify all LPVs in the dwarf galaxies of the Local Group accessible in the northern hemisphere, and then determine the SFHs from their luminosity distribution; obtain accurate time-averaged photometry for all LPVs; obtain the pulsation amplitude of them; determine their radius variations; model their SEDs; and study their mass loss as a function of stellar properties such as mass, luminosity, metallicity, and pulsation amplitude. This is Paper IV in the series, concerned with identifying the LPV stars in And VII and estimating the SFH of this galaxy. The article layout is as follows: the description of the data is given in Section 2. The data reduction and a brief review of the photometry methods are presented in Section 3. The catalog is described in Section 4, followed by the method for finding the LPVs in Section 5. In Section 6, we discuss And VII's CMD and obtain the AGB and RGB tips and the distance modulus. The method of deriving the SFH is described in Section 7. The results and conclusions are explained in Sections 8 and 9, respectively.

\section{Observations}

The data set was obtained with WFC/INT over a period of $3 \mathrm{yr}$ (2015 June to 2017 October) from a survey of the majority of dwarf galaxies in the Local Group, including $43 \mathrm{dSph}$, 6 dIrr, 6 dTrans, and 4 globular clusters, all visible in the northern hemisphere (Saremi et al. 2017, 2020). The WFC is an optical mosaic camera at the prime focus of the $2.5 \mathrm{~m}$ Isaac Newton Telescope (INT) in La Palma, Spain. It consists of four $2048 \times 4096$ CCDs, with a pixel size of 0 ". 33 pixel $^{-1}$. A combined mosaic of And VII from $i$ and $V$ bands is shown in Figure $1\left(11.26 \times 22.55 \operatorname{arcmin}^{2}\right)$.

The survey was designed to measure the amplitude and mean brightness of LPV stars, which are variable owing to radial pulsations on timescales from $\approx 60$ days (McDonald \& Zijlstra 2016) for low-luminosity AGB stars to $\approx 700$ days for intermediate-luminosity AGB stars (Wood et al. 1992), as well as periods up to $\sim 2000$ days for RSGs (Samus' et al. 2006). For this purpose, observations that were spaced a few months apart were taken over eight epochs in the WFC Sloan $i$ filter, except for the first epoch, when the WFC RGO $I$ filter was used and subsequently transformed to Sloan $i$ (explained in Section 3). Additionally, five epochs in the Harris $V$ filter were observed to obtain color information. The details of the observations of And VII are listed in Table 1.

\section{Data Analysis}

Before photometry, the raw CCD frames must be processed. To this end, we used THELI (Transforming HEavenly Light into Image) as an image processing pipeline with astronomical software that is adapted to multichip cameras for the data reduction process (Saremi et al. 2020). Point-spread function (PSF) photometry calibration was performed with the DAOPHOTII package (Stetson 1987) using the DAOPHOT/ALLSTAR/ ALLFRAME routines. Further details, including data processing, photometric calibration, and relative calibration, are given in Saremi et al. (2020).

For obtaining the epochs, we used Sloan $i$ and Harris $V$ filters, except for the first night (2015 June 18), which had another filter (the WFC RGO I filter). Hence, we used transformation equations extracted from Jordi et al. (2006) to estimate magnitudes for all stars in the Sloan $i$ filter. The $R$ band image of 2015 June 18 was used to transform the $I$-band data into Sloan $i$ magnitudes using the following relation:

$$
i-I=(0.251 \pm 0.003) \times(R-I)+(0.325 \pm 0.002) .
$$

Before performing this calculation, it was necessary to determine the zero-point of each frame to provide the ALLFRAME lists in unison. Also, $R$ and $I$ ALLFRAME lists were used to select program stars in the DAOGROW and COLLECT routines to correct the aperture photometry of Sloan $i$ magnitudes, but they have not been used in the NEWTRIAL section. Hereafter, the stellar magnitudes from 


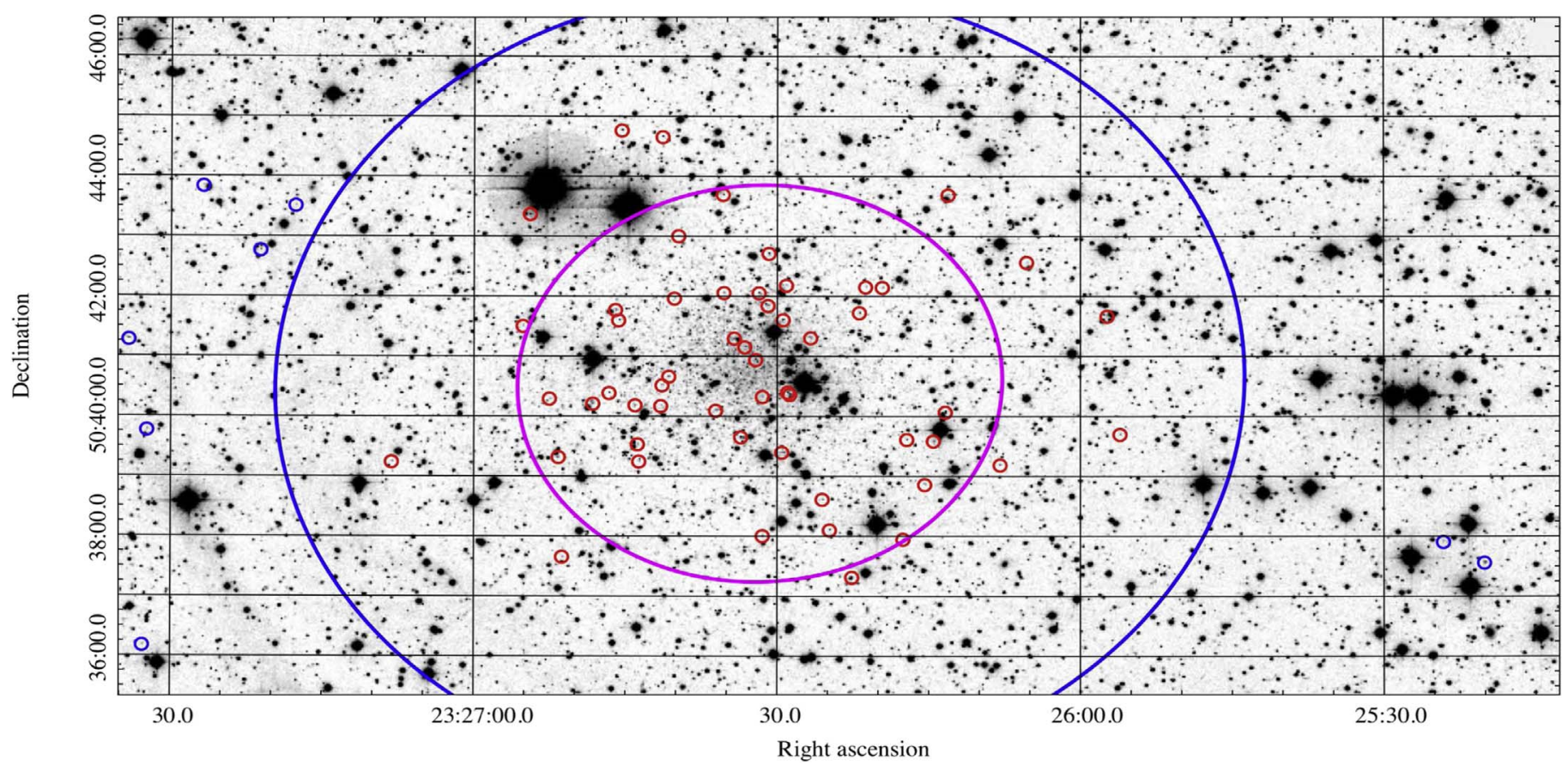

Figure 1. Master WFC image of the And VII dSph galaxy along with the spatial location of LPV candidates (inside $2 r_{\frac{1}{2}}$ in red and outside $2 r_{\frac{1}{2}}$ in blue). The half-light radius is marked with a magenta ellipse $r_{\frac{1}{2}}=3$ '.8 with an ellipticity of 0.13 . The $2 r_{\frac{1}{2}}$ radius is displayed in blue.

Table 1

Log of WFC Observations of the And VII Dwarf Galaxy

\begin{tabular}{|c|c|c|c|c|c|}
\hline $\begin{array}{l}\text { Date } \\
(\mathrm{y} \mathrm{m} \mathrm{d})\end{array}$ & Epoch & Filter & $\begin{array}{l}t_{\exp } \\
(\mathrm{s})\end{array}$ & $\begin{array}{l}\text { Seeing } \\
(\operatorname{arcsec})\end{array}$ & Air Mass \\
\hline 20150618 & 1 & $I$ & 555 & 1.87 & 1.339 \\
\hline 20150618 & 1 & $R$ & 735 & 1.89 & 1.262 \\
\hline 20150618 & 1 & $V$ & 1200 & 1.98 & 1.174 \\
\hline 20160210 & 1 & $i$ & 629 & 2.17 & 1.821 \\
\hline 20160613 & 2 & $i$ & 796 & 3.22 & 2.259 \\
\hline 20160810 & 3 & $i$ & 555 & 2.24 & 1.228 \\
\hline 20160812 & 3 & $i$ & 683 & 2.13 & 1.332 \\
\hline 20160812 & 2 & $V$ & 126 & 2.05 & 1.084 \\
\hline 20161020 & 4 & $i$ & 555 & 2.25 & 1.102 \\
\hline 20170130 & 5 & $i$ & 556 & 1.98 & 1.593 \\
\hline 20170801 & 6 & $i$ & 555 & 1.97 & 1.803 \\
\hline 20170801 & 3 & $V$ & 736 & 2.08 & 1.576 \\
\hline 20170902 & 7 & $i$ & 556 & 2.08 & 1.089 \\
\hline 20170902 & 4 & $V$ & 736 & 2.15 & 1.079 \\
\hline 20171006 & 8 & $i$ & 555 & 2.09 & 1.204 \\
\hline 20171008 & 5 & $V$ & 736 & 2.40 & 1.221 \\
\hline
\end{tabular}

Note. The observation in the $R$ band is used for transforming RGO $I$ to Sloan $i$ band.

all frames are related to two bands (Sloan $i$ and $V$ ) to make the master mosaic of And VII in the final stage of NEWTRIAL.

\subsection{Photometry Evaluation}

The photometric completeness was tested with the ADDSTAR task in the DAOPHOT package; 2500 artificial stars were added to the master mosaic in the $i$ and $V$ bands. These were split over five trials, to avoid increasing the crowding level. The positions of stars were selected randomly in the images, and then Poisson noise was added to them. We specified the magnitude of artificial stars between 16 and 25 mag in 1 mag bins (similar

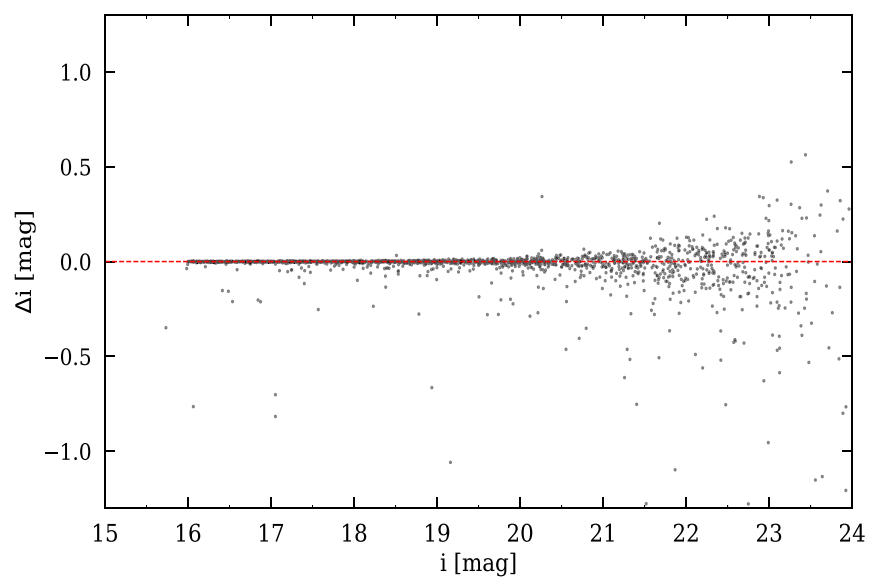

Figure 2. Scatter of difference in magnitudes between the input and the recovered artificial stars.

bins for $V$ band). Then, the photometric reduction was repeated to assess the precision of photometry. Figure 2 shows the difference in magnitudes between the input and the recovered artificial stars using the DAOMASTER routine. The magnitude difference in $i$ band is very small, $|\Delta i|<0.1 \mathrm{mag}$ up to $i \approx 22 \mathrm{mag}$.

Also, we found the fraction of recovered stars in the 0.5 mag bins in both bands to determine the completeness. Figure 3 displays the percent of the recovered stars, which decreases with increasing magnitude and reaches a completeness of 50\% at $i=22.8 \mathrm{mag}$ and $V=23 \mathrm{mag}$. The important range of magnitudes in this project is between the RGB tip ( $i=$ $21.3 \mathrm{mag}, V=22.35 \mathrm{mag})$ and the AGB tip ( $i=17.63 \mathrm{mag}$, $V=17.81 \mathrm{mag}$ ) (see Section 6).

We have performed the photometric calibration process in three steps as explained by Saremi et al. (2020; see also 


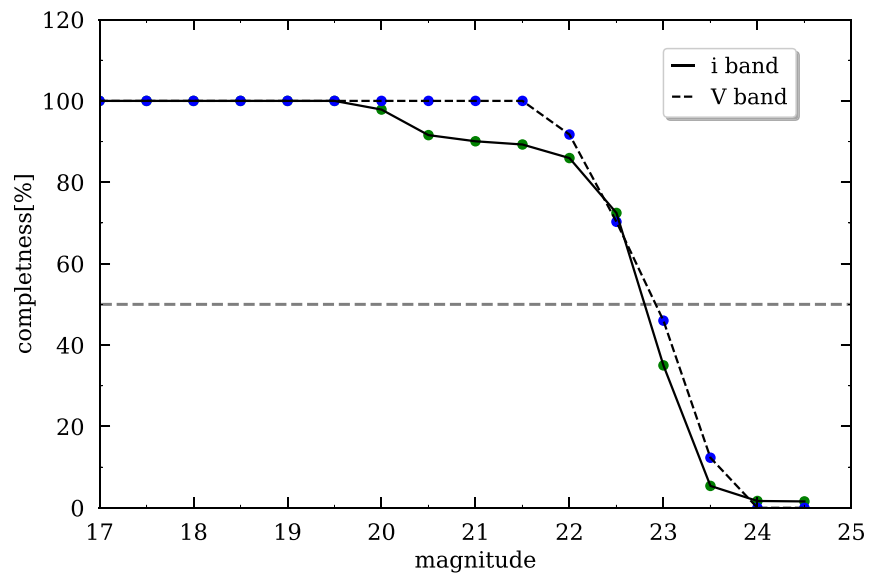

Figure 3. Completeness as a function of $i$-band (solid line) and $V$-band (dashed line) magnitude.

Section 3.2) and obtained the final catalog of 14,092 objects with the NEWTRIAL routine.

\subsection{Calibration}

We have performed the photometric calibration process in three steps:

1. Aperture correction: With the use of the DAOGROW and COLLECT routines, the differences in magnitude between the PSF-fitting and large-aperture photometry of the program stars (20-30 isolated bright stars in each frame) were calculated (Stetson 1990). Finally, NEWTRIAL applied these aperture corrections to all stars for each frame.

2. Transformation to the standard photometric system: The transformation equation for each frame is constructed based on zero-point and atmospheric extinction. Also, for frames without a standard-field observation, we used the mean of other zero-points. The CCDAVE routine applied the transformation equation on the program stars for each frame. Then, program stars as local standards were used in the NEWTRIAL routine to calibrate all the other stars.

3. Relative calibration: Relative photometry achieves a balance between all epochs. Thus, similar magnitudes are obtained for a nonvariable star in all epochs. The calculated relative deviation values for frames are between 0.6 and 9 mmag. The relative deviation of each frame was subtracted from the magnitudes of all stars in that frame. This correctly separates variable from nonvariable sources (Saremi et al. 2020).

\section{Properties of the Data Set}

\subsection{Determining the Half-light Radius of And VII}

Figure 4 shows And VII's surface brightness and stellar number density on concentric elliptical annuli as a function of the semimajor axis in black and red points, respectively. The best-fitting exponential profile is overlaid in blue, using a leastsquares minimization technique. The error bars are derived from the Poisson error on the photon counts. To obtain And VII's half-light radius, $r_{\frac{1}{2}}$, the total extrapolated luminosity of the exponential profile is calculated by integrating up to $r \rightarrow \infty:$

$$
L(r)=2 \pi \epsilon \int_{0}^{r} r S(r) d r
$$

where $S(r)$ is the radial profile with an exponential law and $\epsilon=0.13$ is the measured ellipticity of the galaxy (McConnachie $\&$ Irwin 2006). We thus obtained the semimajor axis of the half-light ellipse, $r_{\frac{1}{2}}=3 ! 8 \pm 0 ! 3$. This agrees with previous values of $3.5 \pm 0$. 1 (McConnachie \& Irwin 2006) and $791 \pm 45 \mathrm{pc}$ corresponding to $3.56 \pm 0$ '.20 (Kalirai et al. 2010).

\subsection{Foreground Contamination}

And VII is located at low Galactic latitude and is contaminated by foreground stars of the Milky Way; hence, disposing of these foreground stars is essential for accurate recognition of LPV stars in the dwarf galaxy. Therefore, we cross-matched our catalog with the second Gaia data release (Gaia DR2; Gaia Collaboration et al. 2018) to remove the contamination of foreground stars in this direction. To enhance the accuracy of foreground star selection, we applied particularly strict criteria on the proper motion and parallax of Gaia stars, which are similar to the criteria of Saremi et al. (2020). Accordingly, 1872 stars were found as foreground stars, but only 380 of them were located within $r_{\frac{1}{2}}$ from the center of And VII. The top left panel of Figure 5 shows the Gaia foreground stars in blue and And VII's candidate members in black for the CCD 4 field. The right panel is similar to the left panel, but for stars located within $r_{\frac{1}{2}}$. The completeness limit of Gaia is near $G \sim 17 \mathrm{mag}$, and unfortunately stars with $i>20.5$ mag are not very well distinguished in this catalog (Gaia Collaboration et al. 2018).

Hence, we also assess the foreground contamination in the direction of And VII and the extinction in the $V$ band of $A_{V}=0.532$ mag, using the TRILEGAL simulation, which reproduces the stellar population artificially (Girardi et al. 2005). In Figure 5, the magenta points represent the predicted contamination of two different fields: $0.07 \mathrm{deg}^{2}$ (about the size of the entire CCD 4 of WFC; left panel) and $0.01 \mathrm{deg}^{2}$ (the half-light radius of And VII; right panel). We compare the number of foreground contaminants with $i<20.5$ mag obtained via two procedures to estimate the accuracy of TRILEGAL prediction. Within $r_{\frac{1}{2}}$, the cross-correlation between our results and Gaia catalog suggested the number 380 foreground stars, which is consistent with the estimation of TRILEGAL simulation (392 stars). In the whole region of CCD 4, 1872 foreground stars are found from the Gaia catalog, while 2664 foreground stars are estimated from the TRILEGAL simulation. However, the $i=20.5 \mathrm{mag}$ is not an exact limit in Figure 5, and decreasing it to $i=20 \mathrm{mag}$, the number of contaminations is reduced to 2152 sources by TRILEGAL and 1763 by Gaia. Therefore, for $i>20.5 \mathrm{mag}$, we can benefit the TRILEGAL simulation and be more sure of estimation in inner regions, which are more important in this study, than outer regions.

Clearly, the region around the galaxy is extremely contaminated by foreground stars, while the CMD of the inner region $\left(r<r_{\frac{1}{2}}\right)$ suffers much less contamination. The measured stellar surface densities within $r_{\frac{1}{2}}$ and across the rest of the field are 0.04 and $0.01 \operatorname{arcsec}^{-2}$, respectively. The TRILEGAL model estimates Milky Way foreground densities over these two regions of $\sim 0.01$ and $\sim 0.02 \operatorname{arcsec}^{-2}$, respectively. Thus, the 


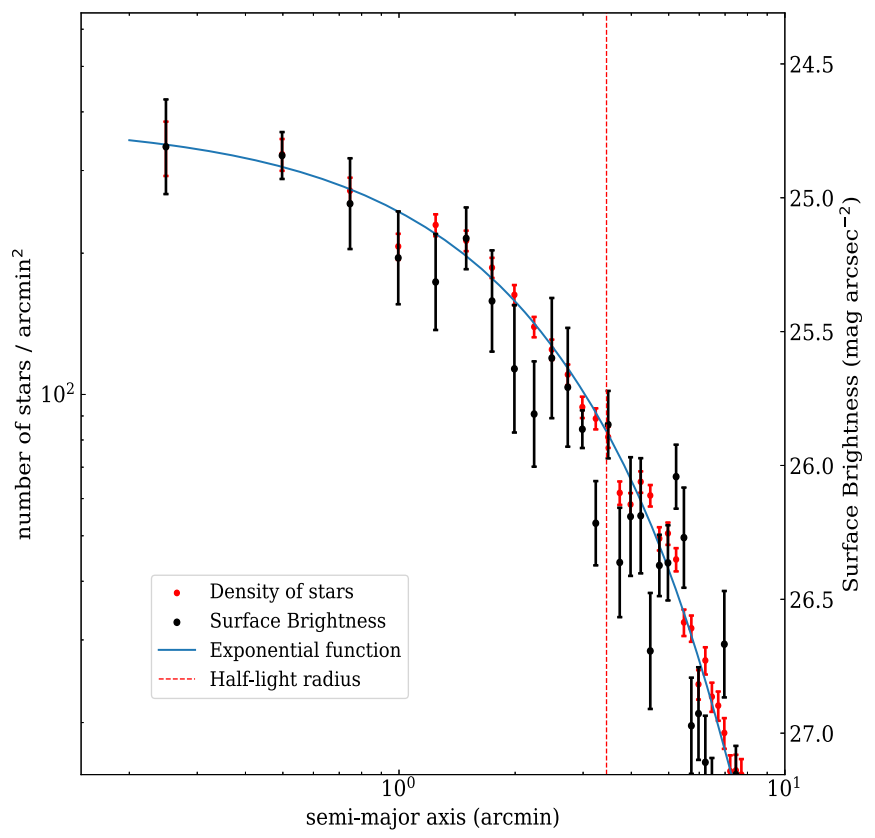

Figure 4. The log-log plot of the stellar number density of And VII is shown in red points, and the surface brightness is shown in black points on the right vertical axis. The blue solid curve depicts the best exponential fit to the data. Error bars are calculated from the Poisson uncertainty in the counts. We determined $r_{\frac{1}{2}}=3 ! 8 \pm 0 ! 3$.

fraction of contaminating foreground stars is much larger outside $r_{\frac{1}{2}}$ than inside. And VII has a large projected distance from M31 (16.2); hence, contamination from M31 halo stars is unlikely to be significant (Tollerud et al. 2012).

\section{Searching for Variable Stars}

In order to identify variable stars, we applied a method similar to the NEWTRIAL program described by Welch \& Stetson (1993) and also developed further by Stetson (1996). This method attributes to each star a variability index $(L)$ that is derived based on $J$ and $K$ indices. Typically, variable stars show a large positive $J$ index, while the $J$ index tends to zero for stars with random noise. Besides, the $K$ index is described based on the shape of the light curve

$$
L=\frac{J \times K}{0.798} \frac{\sum_{i=1}^{N} w_{i}}{w_{\text {all }}},
$$

where $\sum w$ is the total weight assigned to a star and $w_{\text {all }}$ is the total weight that a star would be assigned if observed in all observations. The weight $\left(w_{i}\right)$ of each star is 1 in pairs of frames that are close in time compared to the period of variability (expected $\sim 100$ days or longer), while in a single frame $w_{i}=0.5$.

After having calculated $L$ for all stars, we determined an optimal variability threshold that separates variables from other stars. The histograms of $L$ are presented in four magnitude ranges surrounding the RGB and AGB tips in Figure 6. To determine the variability threshold $\left(L_{\mathrm{var}}\right)$, we mirrored the negative part of each histogram with respect to $L=0$, because we do not expect to have physical variables with $L<0$. Then, a Gaussian function was fitted to these negative parts and their mirrors to resemble the nonvariable sources, allowing us to estimate the percentage of variable stars in each bin. The

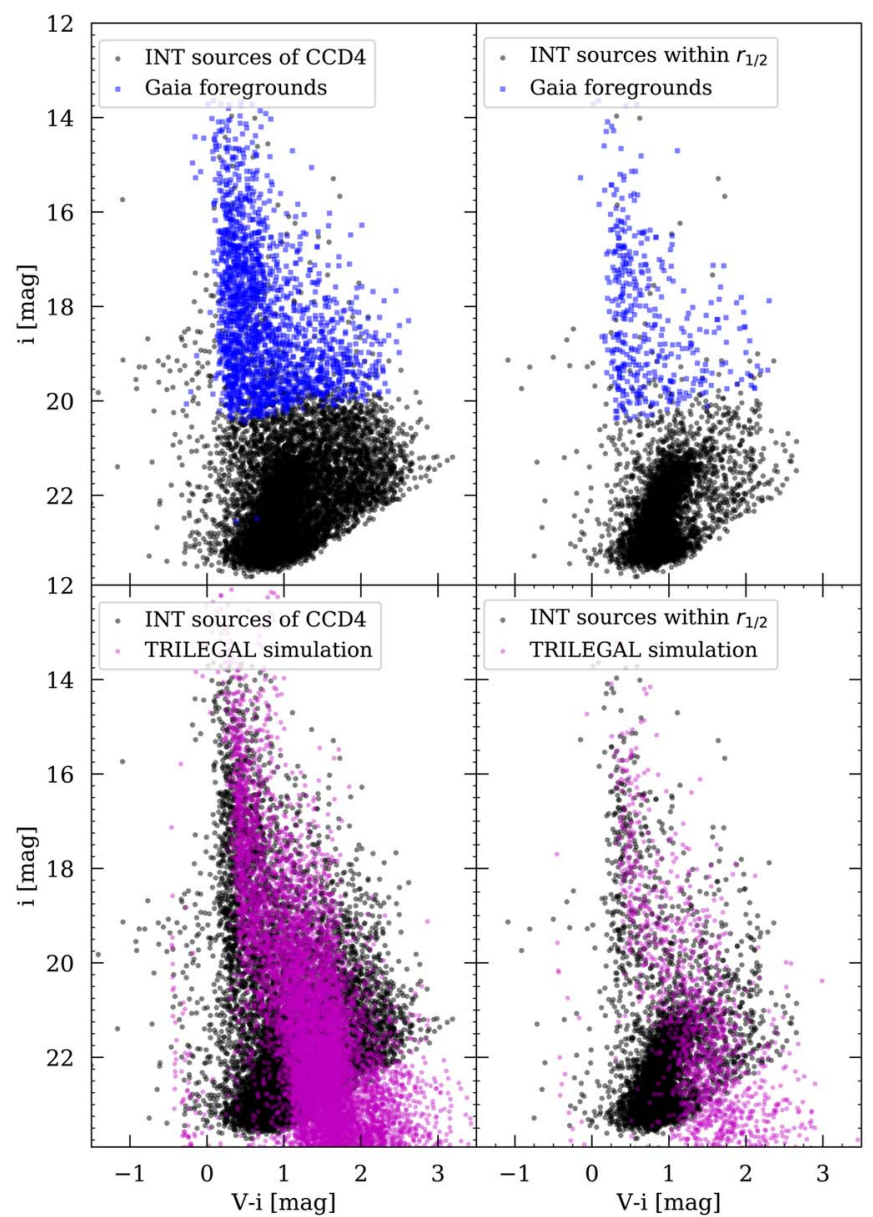

Figure 5. Gaia foreground stars (in blue) and predicted contamination of foreground stars (in magenta) as simulated with TRILEGAL (Girardi et al. 2005) are presented in two panels: the left concerns a $0.07 \mathrm{deg}^{2}$ field, while the right panel is restricted to $0.01 \mathrm{deg}^{2}$ centered on And VII corresponding to the halflight radius of the galaxy. INT sources are in black.

Gaussian fits closely match the histogram at low values of $L$ but deviate from it at higher values, representing the sample of intrinsically variable stars. For each magnitude interval we chose $L_{\mathrm{var}}$ beyond which $90 \%$ of objects in a bin are above the Gaussian fit (i.e., only $10 \%$ of the subset are expected to be nonvariable). The threshold values of $L$ are labeled in Figure 6.

In Figure 7, we show the variability index of all stars versus magnitude, along with a polynomial curve fit to the threshold points obtained from histograms. $L_{\mathrm{var}}$ increases with $i$-band magnitude, as the photometric uncertainties increase. The red stars with $L>L_{\mathrm{var}}$ in the plot are candidate variables if they are not visibly affected by blending. After eliminating these suspect stars, we identified 154 candidate variable stars in the CCD field, most of which are between $i \approx 20$ and $22.5 \mathrm{mag}$, i.e., around the RGB tip.

\subsection{Amplitudes of Variability}

Figure 8 shows the light curve of a nonvariable star (in the top panel), and two light curves of candidate LPV stars labeled with green points in Figure 9 are presented in the middle and bottom panels. The amplitude of the light curve can be estimated by assuming a sinusoidal shape. The rms of a sine wave with unit amplitude is 0.701 , so the amplitude of a light curve with a standard deviation of $\sigma$ can be calculated from 

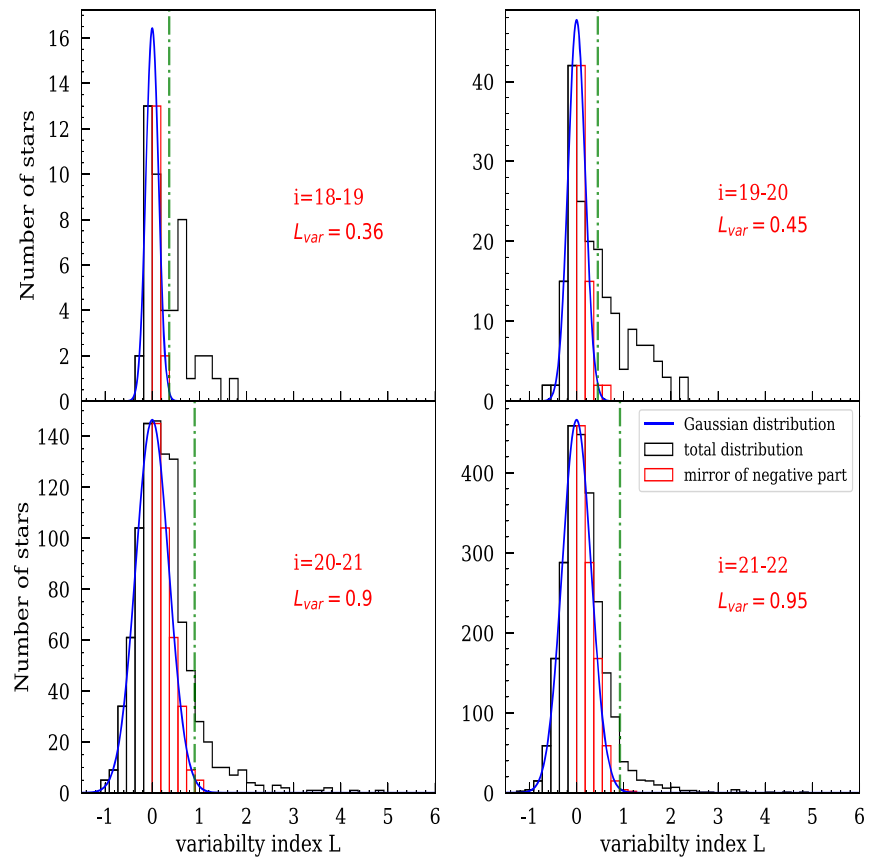

Figure 6. Histograms of the variability index $L$, for several $i$-band magnitude bins. The negative part of each histogram is mirrored (red bins). This is fitted with a Gaussian function to show the expected distribution of nonvariable sources. The optimal variability index thresholds are indicated by vertical green dashed lines.

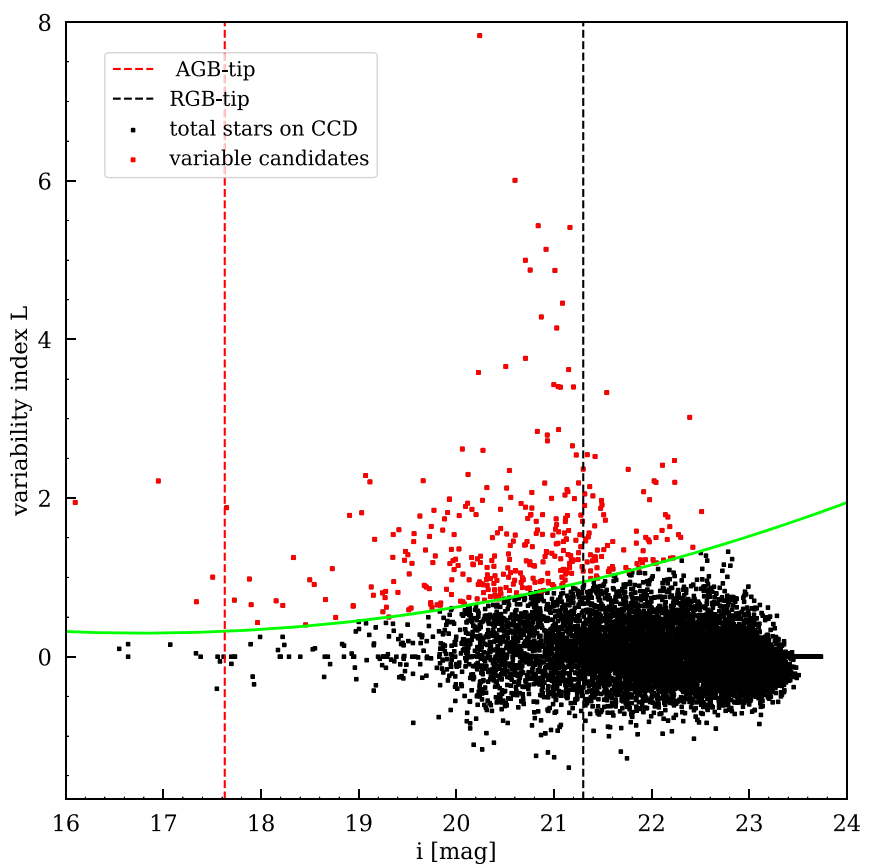

Figure 7. Variability index $L$ of all stars vs. $i$-band magnitude. The green curve indicates the threshold for different magnitudes. As a reminder, positively identified foreground stars have already been removed.

$A=2 \sigma / 0.701$. We might not detect the faint or large-amplitude variables in some epochs when they drop below our detection threshold. In this case, we will underestimate their $i$-band variability.

The $i$-band amplitude of candidate LPV stars versus their $i$-band magnitude is shown in the left panel of Figure 9, and the amplitude versus their $(V-i)$ color is presented in the right panel. A distinct feature of the left panel is that LPV amplitudes
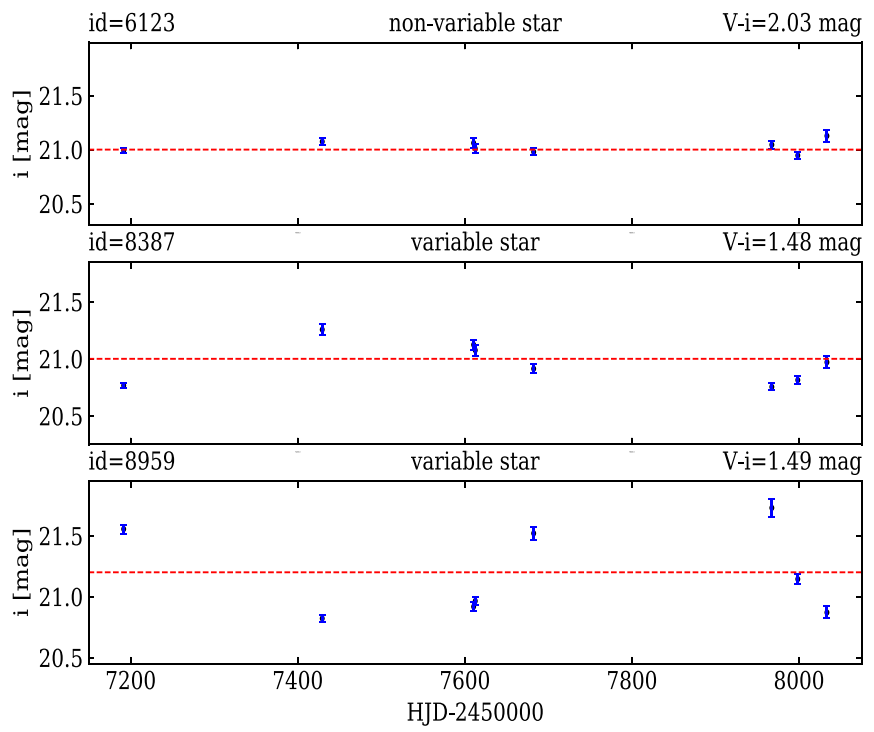

Figure 8. Light curves of two LPV candidates labeled with green color in Figure 9, and a nonvariable star in the top panel for comparison.
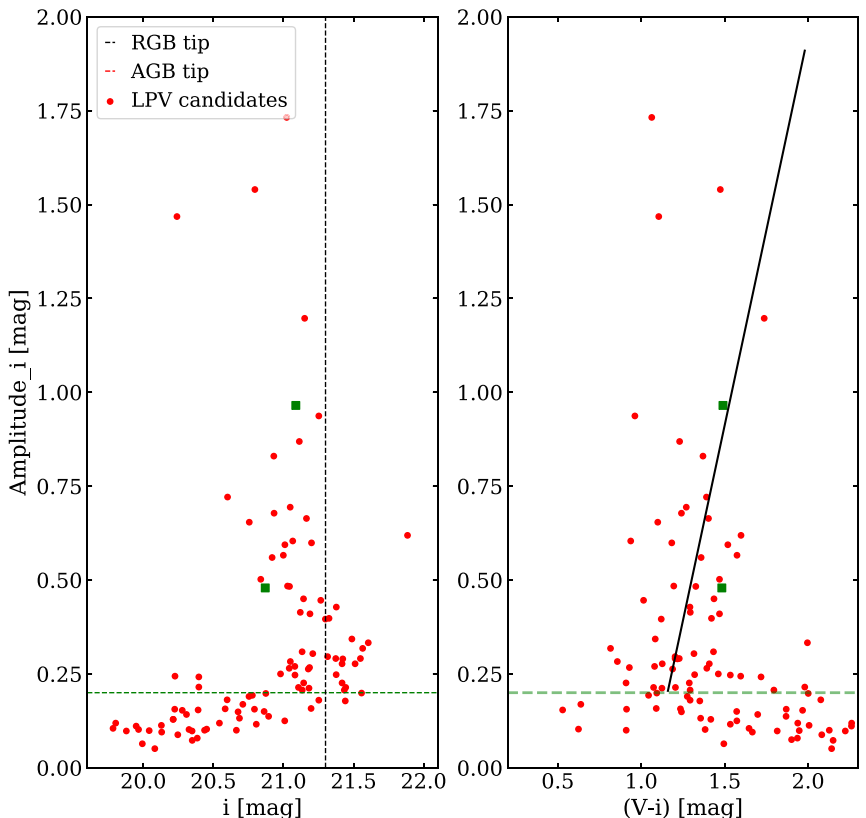

Figure 9. Estimated amplitude of variability $A_{i}$ vs. $i$-band magnitude (left panel) and $(V-i)$ color (right panel). The green dashed horizontal lines separate variables with $A_{i}<0.2 \mathrm{mag}$. The light curves of the green points are displayed in Figure 8. The RGB tip is indicated by the black dashed line in the left panel (see Section 6), and the solid black line shows the least-squares fit in the right panel. There is a high-amplitude source with $A_{i}=3.78$ and color $(V-i)=1.64$ that falls outside the boundaries of the graph.

increase with decreasing brightness until $A_{i} \sim 1.8 \mathrm{mag}$ (Navabi et al. 2020). In the right panel, the weighted average of colors for three different bins, $0.2 \mathrm{mag}<A_{i}<0.5 \mathrm{mag}$, $0.5 \mathrm{mag}<A_{i}<1 \mathrm{mag}$, and $A_{i}>1 \mathrm{mag}$, are $1.21 \pm 0.03 \mathrm{mag}$, $1.33 \pm 0.05 \mathrm{mag}$, and $1.61 \pm 0.12 \mathrm{mag}$, respectively. Thus, it shows that LPV stars with larger amplitudes generally become redder. Also, the solid black line represented in the right panel shows this trend by the weighted least-squares fitting for points with $A_{i}>0.2$. These trends seen in And VII are like those seen before in And I (Saremi et al. 2020). As shown in Figure 9, a number of candidate variable stars have $A_{i}<0.2 \mathrm{mag}$, and we 


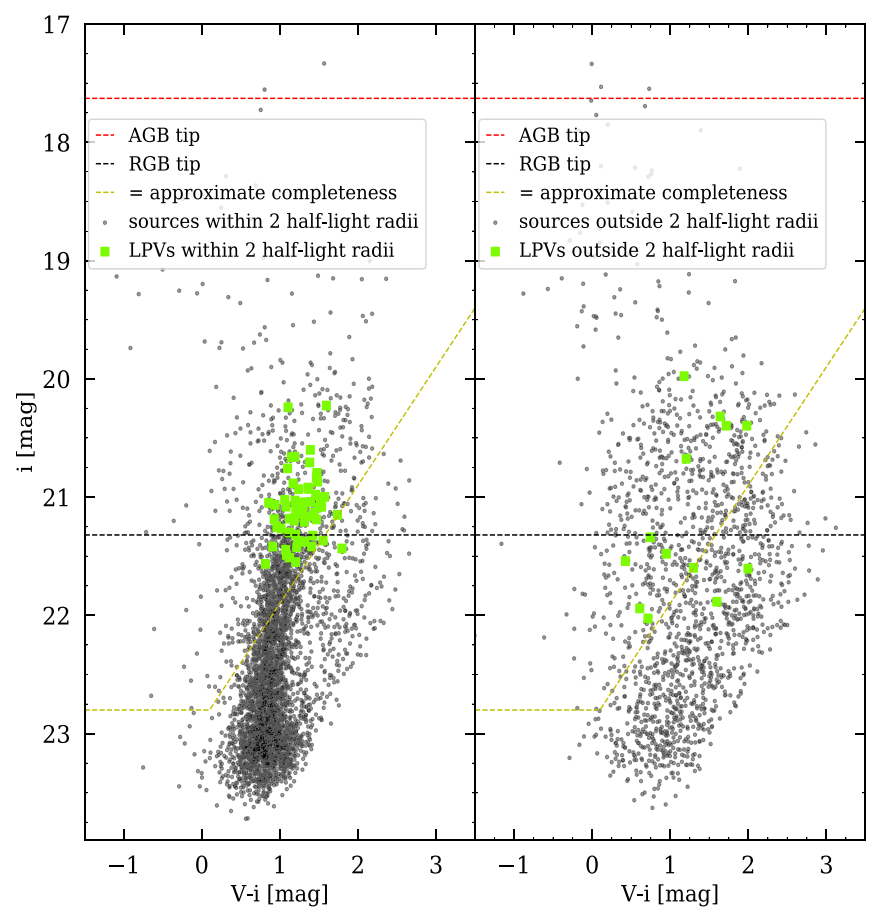

Figure 10. CMD of And VII, showing our identified candidate LPV stars, in green, for two regions with similar areas: within two half-light radii (left panel) and outside two half-light radii (right panel). The latter shows mainly the contamination from foreground (and background) sources.

cannot be certain about the nature of their variability. Therefore, we only consider high-amplitude variable stars as candidate LPV stars.

In the entire field of CCD 4, 62 candidate LPV stars have been identified, of which 43 are inside $r_{\frac{1}{2}}$ from the center of And VII. The density of candidate LPV stars inside $r_{\frac{1}{2}}$ is $1.1 \operatorname{arcmin}^{-2}$, which is much larger than the density of 0.03 arcmin $^{-2}$ outside this radius; hence, we can trust that most detected candidate LPV stars truly belong to And VII. Figure 1 shows the positions of the $55 \mathrm{LPV}$ candidates within $2 r_{\frac{1}{2}}$ in red and 7 out of the remaining 62 LPV candidates in blue, covering a similar area on the sky. Additionally, Figure 10 shows the CMDs of these two regions (left and right panels for inside and outside $r_{\frac{1}{2}}$ ) with candidate LPV stars in green and other sources in black. By comparing the two CMDs, we can estimate the total contamination from background galaxies and active galactic nuclei and foreground stars that are below the limit of completeness of Gaia. Hence, within $2 r_{\frac{1}{2}}$ of the center of And VII, $\sim 46 \%$ of all stars and only $16 \%$ of candidate LPV stars could be contaminants. The lack of a curved RGB outside $r_{\frac{1}{2}}$ (right panel of Figure 10) supports the notion that contamination from M31 is insignificant; hence, the Milky Way remains the major source of contamination.

\section{Color-Magnitude Diagram}

Figure 11 presents the CMD of $\sim 10,000$ stars restricted to the $2 r_{\frac{1}{2}}$ ellipse of And VII, with 55 candidate LPV stars in green. The overplotted Padova PARSEC/COLIBRI isochrones (Bressan et al. 2012; Marigo et al. 2017; Pastorelli et al. 2019) were computed using the CMD v3.3 interface, ${ }^{15}$ adopting a

\footnotetext{
15 http://stev.oapd.inaf.it/cgi-bin/cmd_3.3
}

metallicity of $Z=0.0007$, and covering a range of masses between 0.15 and $120 M_{\odot}$. This model is derived for over 50 different photometric systems and instruments, including SDSS and Landolt, and predicts the large-amplitude pulsation, the related mass-loss rate, and the dust formation rate. Also, the $50 \%$ completeness limit of our photometry is determined based on a simple simulation in Section 3.1. The CMD demonstrates an early-type galaxy, with a prominent RGB branch and no evidence for young blue stellar sequences, but with a small intermediate-age AGB population immediately above it, dominated by our candidate LPV stars.

To quantify the AGB population, we must now define the magnitudes of the RGB and AGB tips, which are determined by the brightest star belonging to the RGB and AGB. In the RGB case, this corresponds to the start of helium burning. However, the AGB tip in this color combination does not represent the end of $\mathrm{AGB}$ evolution, but the point where the color-luminosity relationship breaks down owing to circumstellar dust production, resulting in the optical obscuration of the stars. The RGB tip can be used as a standard candle, since the RGB luminosity distribution shows a discontinuity. To obtain the RGB tip, the $I$ band is most suitable, as it exhibits minimal dependence on age and chemical composition (Lee et al. 1993). Therefore, we transformed the Sloan $i$ magnitudes to the $I$ band of the Johnson-Cousins system using the transformations from Lupton (2005). RGB stars are selected as those inside $r_{\frac{1}{2}}$ if they are located between the two blue lines on the CMD of Figure 12. Then, the luminosity probability distribution (LPD) was derived from them. The LPD technique is appropriate because there are a sufficient number of RGB stars and the effect of Poisson noise is modest (McConnachie et al. 2004).

We show both the binned luminosity histogram of $I$ magnitudes (with 0.05 mag bins) and also the LPD (curved plot) in the middle panel of Figure 12. The right panel presents the convolution of the LPD with the Sobel kernel $[-2,-1,0$, $1,2]$. The peak of this response is marked by the red dashed line, which we identify as the RGB tip in the $I$ band $(20.7 \pm 0.05 \mathrm{mag})$, from which we derive a distance modulus for the system of $\mu=24.38 \pm 0.05$ mag with the adopted value of $M_{I}^{\mathrm{TRGB}}$ and extinction from McConnachie et al. (2004). This result is in accordance with earlier studies (see Section 1). We also obtained a magnitude for the RGB tip in the $i$ band of $21.3 \pm 0.05$ mag based on transforming again to Sloan $i$, which is shown in Figures 10 and 11 as a black dashed line.

We used the brightest point of isochrones to determine the theoretical AGB tip in the CMD (see Saremi et al. 2020). The $M_{\text {bol }}$ for a Chandrasekhar core mass is -7.1 mag, based on the classical core luminosity relation. This core luminosity is reached by stars with $\log (t / \mathrm{yr}) \lesssim 7.55$ in the adopted Padova isochrones. Accordingly, the peak of the $\log (t / \mathrm{yr}) \sim 7.55$ isochrone reaches $i=17.63 \mathrm{mag}$ for an adopted metallicity of $Z=0.0007$ and distance modulus $\mu=24.38 \mathrm{mag}$, which is shown by the red dashed line on the CMD of Figure 11. Besides, the Padova PARSEC/COLIBRI isochrones produce carbon stars in the range $\log (t / \mathrm{yr})=8.0-9.4$ and $M_{\text {init }}=1.3-4.9 M_{\odot}$. The limit to carbon star masses is set by the efficiency of third dredge-up, which influences both when a carbon star is formed and when hot-bottom burning prevents a star from becoming carbon-rich.

Studies of carbon AGB stars in M31 dSph companions have recently been performed to map the extent of intermediate-age populations. Harbeck et al. (2004) found that most carbon stars 


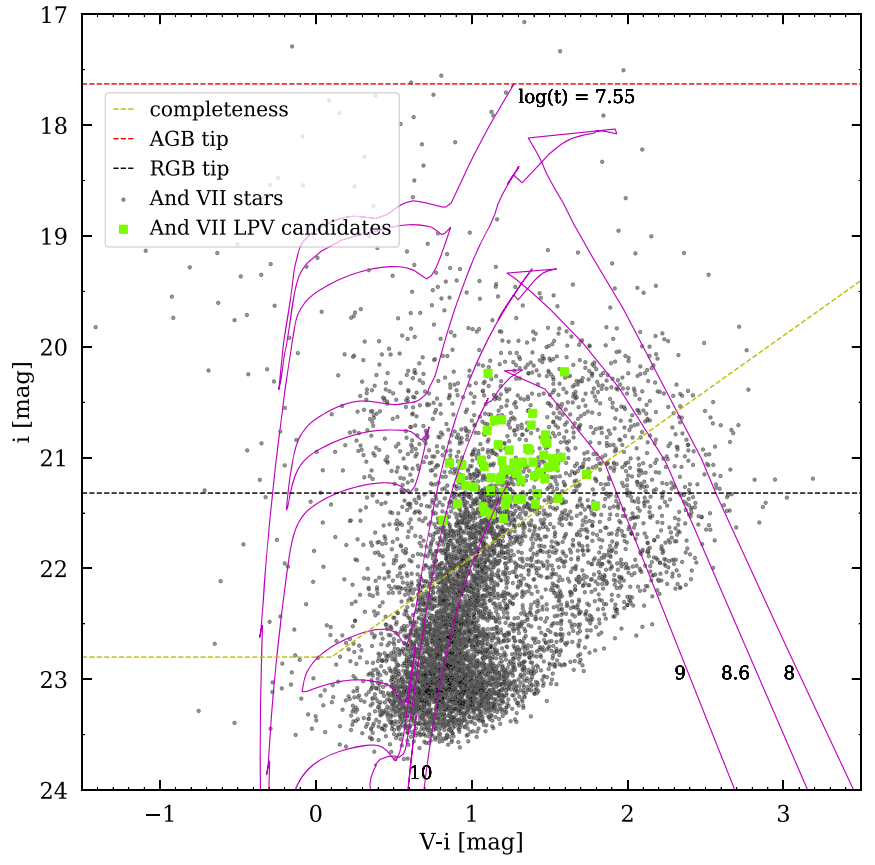

Figure 11. CMD of And VII with candidate LPV stars labeled in green color. The CMD is overlain by the Padova stellar evolution models, and the AGB tip and RGB tip are illustrated by the red and black dashed lines, respectively. The $50 \%$ completeness limit of our photometry is indicated with a yellow dashed line.

reside in And VII among all M31 dwarf galaxies, so they discussed the presence of a substantial intermediate-age stellar population in And VII. To detect red giant stars with enhanced carbon abundance, they used two narrowband filters centered on the $\mathrm{TiO}$ and $\mathrm{CN}$ features at 778 and $808 \mathrm{~nm}$. Carbon stars were identified on the basis of $\mathrm{CN}-\mathrm{TiO} \geqslant 0.2$ and $V-I>1.8 \mathrm{mag}$ and also a luminosity condition that carbon stars obey $I>22 \mathrm{mag}$. Hence, they found in And VII three genuine carbon stars whose bolometric luminosities are brighter than the RGB tip and two carbon stars with dimmer luminosities than the RGB tip.

The five carbon candidates from Harbeck et al. (2004) are detected in the INT survey and identified in the CMD of Figure 12 by red points. One of them is shown in green, since we also detect it as a candidate LPV star. All of them exhibit $(V-i)>1.8 \mathrm{mag}$ in the INT survey, likely indicating reddening due to circumstellar dust. The presence of carbon stars with lower luminosity than the RGB tip can be related to mass transfer from an erstwhile carbon star companion, or they may be intrinsic carbon stars at low metallicity (Hamren et al. 2016). These stars are observed in other metal-poor galaxies and globular clusters, e.g., $\omega$ Centauri (van Loon et al. 2007; McDonald et al. 2011) or Sagittarius dwarf irregular galaxy (McDonald et al. 2012). It is worth mentioning that the DUSTiNGS star/extreme-AGB star catalog (Boyer et al. 2015) did not report any variable AGB stars in And VII. The reason is likely that two epochs are insufficient to catch all variability.

\section{Star Formation History}

To reconstruct the SFH, we compare our photometric data to the Padova models described in Section 6. LPV stars are assumed to be at their very ultimate point of evolution and
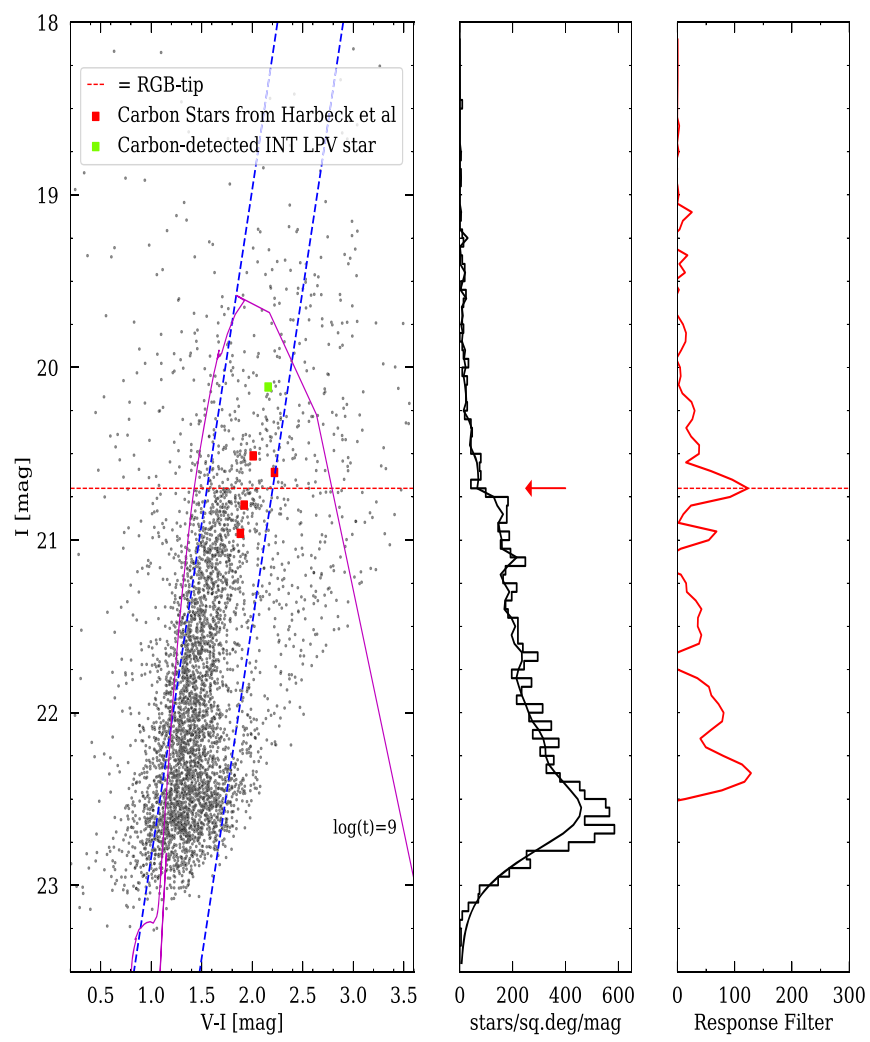

Figure 12. And VII CMD (left panel), luminosity function and LPD (middle), and response filter (right). The RGB tip is measured at $I=20.7$ mag and marked on the CMD by a horizontal red dotted line and on the luminosity functions by an arrow. The left diagram shows the carbon stars from the Harbeck et al. (2004) survey that were detected in our INT survey. A 1 Gyr isochrone is shown for comparison.

to have reached their maximum luminosity. Thus, their brightness can be turned into their birth mass by applying theoretical evolutionary tracks. Hence, a mass-magnitude relationship was defined for the $i$ band by the brightest points on the Padova isochrones. This relationship is displayed in the left panel of Figure 13 for isochrones with an age range of $6.5<\log (t / \mathrm{yr})<10.16$, with step size $\log (t / \mathrm{yr})=0.05$.

It is clear that there is a mostly linear relationship between mass and $i$-band magnitude. However, in the mass range $0.6<\log \left(M / M_{\odot}\right)<0.9$, an inversion appears as a result of the change in atmospheric composition, with oxygen-rich stars occurring during this mass range owing to hot-bottom burning, while the carbon stars below this mass range have brighter $i$ band magnitudes owing to the different molecular opacities between $\mathrm{TiO}$ and $\mathrm{C} \_2 / \mathrm{CN}$. Hence, the mass-magnitude relation was interpolated over this mass range, and the bestfitting function on the other points was obtained with the IRAF task NF-displayed by red lines in Figure 13. The coefficients and intercepts for this mass-magnitude relation are recorded in Table 2. Accordingly, we can estimate the birth mass of LPV stars from this theoretical relation $\left(\log M / M_{\odot}=a \times i+b\right)$.

Furthermore, the relations between birth mass and age and also between birth mass and pulsation duration are shown in the middle and right panels of Figure 13, respectively. The coefficients of the linear fits for mass-age and a set of five Gaussian fittings for mass-pulsation duration are listed in Tables 3 and 4 , for a metallicity of $Z=0.0007$ (relations for 

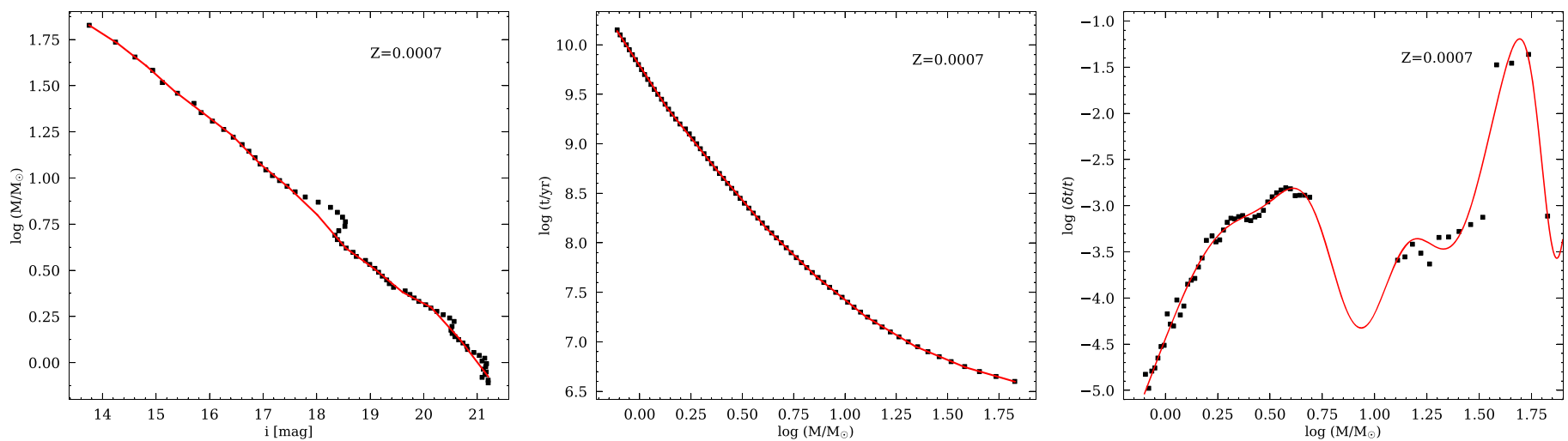

Figure 13. Relation between birth mass and $i$-band magnitude at the end points of stellar evolution for And VII, for a distance modulus of $\mu=24.38$ mag, interstellar attenuation $A_{i}=0.36 \mathrm{mag}$, and metallicity $Z=0.0007$. Solid red lines are fits, in which the function is interpolated over the super-AGB phase $\left(0.6<\log \left(M / M_{\odot}\right)<0.9\right)$.

Table 2

Fitted Coefficients for the Relation between Birth Mass and $i$-band Magnitude, $\log M / M_{\odot}=a \times i+b$ for a Distance Modulus of $\mu=24.38$ mag and a Metallicity $Z=0.0007$

\begin{tabular}{ccc}
\hline \hline$a$ & $b$ & Validity Range \\
\hline$-0.186 \pm 0.063$ & $4.383 \pm 0.922$ & $i \leqslant 14.285$ \\
$-0.227 \pm 0.064$ & $4.968 \pm 0.967$ & $14.285<i \leqslant 14.817$ \\
$-0.260 \pm 0.062$ & $5.461 \pm 0.972$ & $14.817<i \leqslant 15.349$ \\
$-0.221 \pm 0.055$ & $4.863 \pm 0.879$ & $15.349<i \leqslant 15.882$ \\
$-0.223 \pm 0.047$ & $4.889 \pm 0.786$ & $15.822<i \leqslant 16.414$ \\
$-0.296 \pm 0.042$ & $6.085 \pm 0.714$ & $16.414<i \leqslant 16.946$ \\
$-0.239 \pm 0.042$ & $5.124 \pm 0.737$ & $16.946<i \leqslant 17.478$ \\
$-0.277 \pm 0.094$ & $5.799 \pm 1.724$ & $17.478<i \leqslant 18.010$ \\
$-0.338 \pm 0.091$ & $6.892 \pm 1.699$ & $18.010<i \leqslant 18.542$ \\
$-0.210 \pm 0.037$ & $4.520 \pm 0.710$ & $18.542<i \leqslant 19.075$ \\
$-0.240 \pm 0.037$ & $5.081 \pm 0.727$ & $19.075<i \leqslant 19.607$ \\
$-0.155 \pm 0.039$ & $3.418 \pm 0.803$ & $19.607<i \leqslant 20.139$ \\
$-0.322 \pm 0.034$ & $-6.522 \pm 0.698$ & $20.139<i \leqslant 20.671$ \\
$-0.382 \pm 0.027$ & $-8.165 \pm 0.572$ & $i>20.671$ \\
\hline
\end{tabular}

Table 3

Relation between Age and Birth Mass, $\log t=a \log M / M_{\odot}+b$, for a Metallicity $Z=0.0007$

\begin{tabular}{ccc}
\hline \hline$a$ & $b$ & Validity Range \\
\hline$-3.189 \pm 0.024$ & $9.788 \pm 0.006$ & $\log M \leqslant 0.133$ \\
$-2.594 \pm 0.022$ & $9.709 \pm 0.011$ & $0.133<\log M \leqslant 0.375$ \\
$-2.441 \pm 0.023$ & $9.652 \pm 0.017$ & $0.375<\log M \leqslant 0.617$ \\
$-2.002 \pm 0.025$ & $9.382 \pm 0.025$ & $0.617<\log M \leqslant 0.859$ \\
$-1.680 \pm 0.028$ & $9.105 \pm 0.034$ & $0.859<\log M \leqslant 1.101$ \\
$-1.248 \pm 0.032$ & $8.629 \pm 0.047$ & $1.101<\log M \leqslant 1.343$ \\
$-0.867 \pm 0.037$ & $8.118 \pm 0.064$ & $1.343<\log M \leqslant 1.585$ \\
$-0.601 \pm 0.045$ & $7.696 \pm 0.088$ & $\log M>1.585$ \\
\hline
\end{tabular}

other metallicities used in this paper can be found in Figure 19 and Tables 7-9).

\subsection{Method of Deriving the SFH}

The method used for deriving the SFH was developed by Javadi et al. (2011a, 2011b, 2017) and also applied and justified extensively by Rezaeikh et al. (2014), Hamedani Golshan et al. (2017), and Hashemi et al. (2019). The SFR is described as the rate at which gas is transformed into stars, $\xi$ (in $M_{\odot} \mathrm{yr}^{-1}$ ), as a function of time. The amount of stellar mass, $d M$, created in a
Table 4

Fits of the Relation between Relative Pulsation Duration $(\delta t / t$, where $t$ is the Age and $\delta t$ is the Pulsation Duration) and Birth Mass, $\log (\delta t / t)=\Sigma_{n=1}^{5} a_{n} \exp \left[-\left(\log M\left[M_{\odot}\right]-b_{n}\right)^{2} / c_{n}^{2}\right]$, for a Metallicity $Z=0.0007$

\begin{tabular}{lccc}
\hline \hline$i$ & $a$ & $b$ & $c$ \\
\hline 1 & -82861.55 & 1.033584 & 0.345593 \\
2 & 12.84194 & 0.769341 & 0.271284 \\
3 & -8.071388 & -0.878686 & 1.132971 \\
4 & -3.383325 & 1.874786 & 0.111842 \\
5 & 82852.97 & 1.033613 & 1.033613 \\
\hline
\end{tabular}

time interval $d t$ is

$$
d M(t)=\xi(t) d t .
$$

To describe the SFH, we used the LPV stars identified in And VII and their luminosity distribution function, $f(i)$, to reconstruct the birth mass function from the present stellar masses $(M)$. Hence, the SFR as a function of look-back time $(t)$ is

$$
\xi(t)=\frac{f(i(M(t)))}{\delta(M(t)) f_{I M F}(M(t))},
$$

where $\delta$ is the duration of the evolutionary phase during which LPV stars demonstrate strong radial pulsation and $f_{\text {IMF }}$ is the initial mass function (IMF) that defines the proportional SFR of stars of different mass. Each of these functions is related to the stellar mass $(M)$, and the mass of a pulsating star at the end of its evolution is directly associated with its age (Javadi et al. 2011b). With $f_{\text {IMF }}$ defined by

$$
f_{\mathrm{IMF}}=A m^{-\alpha},
$$

where $A$ is the normalization constant and $\alpha$ depends on the mass range, following Kroupa (2001):

$$
\alpha=\left\{\begin{array}{lc}
+0.3 \pm 0.7, \text { if } & 0.02<\frac{m}{M_{\odot}}<0.08 \\
+1.3 \pm 0.5, \text { if } & 0.08<\frac{m}{M_{\odot}}<0.5 \\
+2.3 \pm 0.3, \text { if } & 0.5<\frac{m}{M_{\odot}}<200 .
\end{array}\right.
$$

There is convincing empirical evidence that LPV stars start to lose their mass and produce dust in this phase (e.g., 
Tej et al. 2003; Zhao-Geisler et al. 2012; Ohnaka et al. 2017). The bending of isochrones after their peak shows the evolution of dusty LPV stars that become dimmer and redder. Therefore, dereddening correction for color and magnitude must apply to each LPV star that is under the effect of high dust column density. The magnitude correction in the $i$ band was obtained for the dusty LPV candidates on the CMD (Figure 11) to return them to the expected magnitude (peak of isochrones). To this end, the slopes of $\log (t / \mathrm{yr}) \simeq 9$ isochrones are used for carbon stars, and isochrones of $\log (t / \mathrm{yr})=10$ or 8 are employed for $\mathrm{M}$ stars. The average slopes of isochrones were derived as $a_{\text {oxygen }}=2.04$ if $i \leqslant 20.05 \mathrm{mag}$, and otherwise $a_{\text {oxygen }}=2.95$, and also $a_{\text {carbon }}=1.37$ if $i \leqslant 20.87 \mathrm{mag}$, and otherwise $a_{\text {carbon }}=3.65$. We applied the following correction equation for stars that have $(V-i)>1.4 \mathrm{mag}$ :

$$
i_{0}=i+a\left((V-i)_{0}-(V-i)\right),
$$

where the peaks of all isochrones are located at $(V-i)_{0}=1.16 \mathrm{mag}$. We determined the type of star based on its mass: the mass range of $1.3<M / M_{\odot}<4$ comprises carbon stars, and below or above this range comprises oxygen stars. Among 55 candidate LPV stars, the presence of 16 carbon stars was estimated, out of which one carbon LPV star had previously been found by Harbeck et al. (2004) (Figure 12). The selected range for the mass of carbon stars is examined further in Section 8.3.

To derive the SFH, we followed the procedure described by Javadi et al. (2011b):

1. Using the $i$-band carbon attenuation correction equation for each LPV's magnitude, we obtain the original luminosity. Then, if the mass is not between 1.1 and $4 M_{\odot}$, we deredden with oxygen correction.

2 . Using the corrected $i$-band magnitude and mass-magnitude relation in Table 2, the birth mass is determined.

3. Using the age-mass relation in Table 3, the age is determined.

4. Using the pulsation duration-mass relation in Table 4, the pulsation duration of long-period variability is determined.

5. Applying Equations (4) and (5), we calculate the SFR for selected age bins that have the same number of LPV stars.

6. Using Poisson statistics, we calculate the statistical error for each bin (Hamedani Golshan et al. 2017).

\section{Results and Discussion}

The estimated SFH of And VII within $2 r_{\frac{1}{2}}$ and $r_{\frac{1}{2}}$ of the galaxy's center for different constant metallicities, over an interval from $13.8 \mathrm{Gyr} \quad(\log t=10.14)$ to $\sim 1 \mathrm{Gyr}$ ago $(\log t=9)$, is presented in Figures 14 and 15, respectively. The SFH is characterized by bins that have lengths indicated by horizontal error bars; the vertical error bars show the statistical errors on the SFR in each bin. As we expect for dSph galaxies, star formation continued up to a specific time (perhaps infalling time), and after that, star formation appears to have stopped entirely. For And VII, star formation continued up until 1 Gyr ago, and the SFH shows evidence of an intermediate-age stellar population.

Assuming $Z=0.0007$ within $2 r_{\frac{1}{2}}$, the peak of star formation occurred $\sim 6.2 \mathrm{Gyr}$ ago, when the SFR reached a level of $0.0044 \pm 0.0018 \mathrm{kpc}^{-2}$ on average over an interval of $\sim 0.2$ Gyr. This is at least 12 times as high as the next three

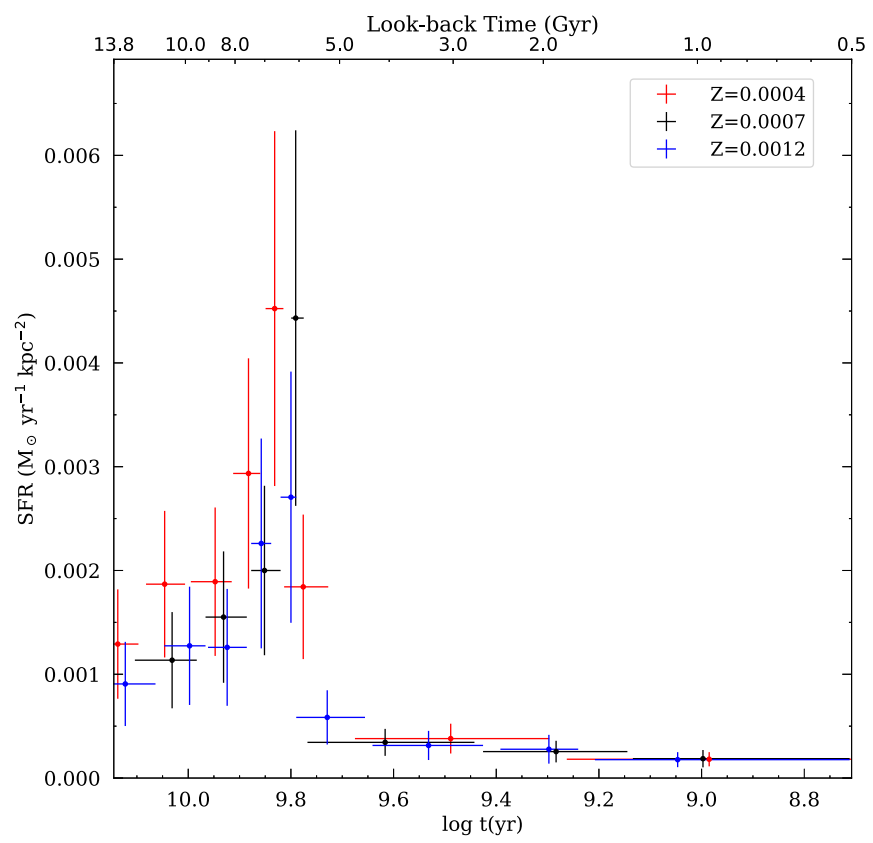

Figure 14. SFH of And VII within $2 r_{\frac{1}{2}}$ for three assumed (constant) metallicities of $Z=0.0004,0.0007$, and 0.0012 in red, black, and blue color, respectively.

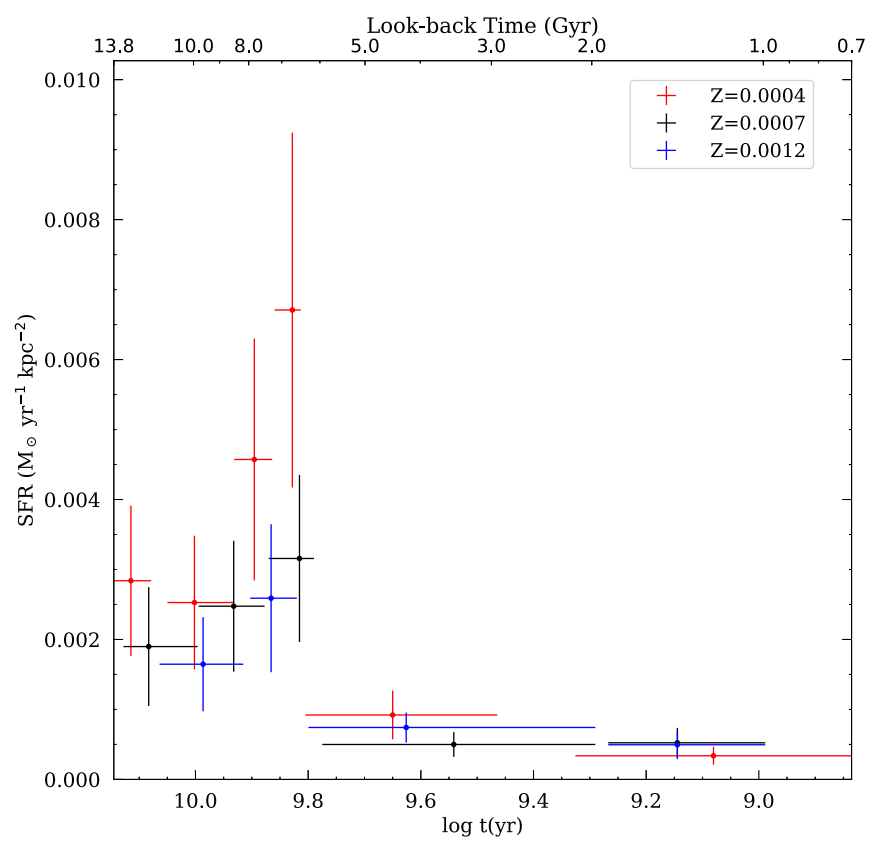

Figure 15. SFH of And VII within $r_{\frac{1}{2}}$ for three assumed (constant) metallicities of $Z=0.0004,0.0007$, and 0.0012 in red, black, and blue color, respectively.

epochs between 6 and 1 Gyr ago. Approximately $90 \%$ of the total stellar mass $\left(13.9 \times 10^{6} M_{\odot}\right)$ in And VII was formed during the period of intense star formation from 13.8 to $6 \mathrm{Gyr}$ ago. After the peak, the SFR dropped suddenly and continued at a rate of $\simeq 0.0003 \pm 0.0002 M_{\odot} \mathrm{yr}^{-1} \mathrm{kpc}^{-2}$ until $1 \mathrm{Gyr}$ ago. However, in the central part $\left(r \leqslant r \frac{1}{2}\right)$ the SFR peaked at a lower rate, $0.003 \pm 0.001 M_{\odot} \mathrm{yr}^{-1} \mathrm{kpc}^{-2}$ at $\log t(\mathrm{yr}) \sim 9.8$.

The total stellar mass $\left(M_{\mathrm{tot}}\right)$ formed inside $r_{\frac{1}{2}}$ and $2 r_{\frac{1}{2}}$ was estimated to be $(13.3 \pm 5.3) \times 10^{6} M_{\odot}$ and $(15.4 \pm 6.2) \times$ $10^{6} M_{\odot}$, respectively (Table 5). These are in agreement with the 
Table 5

Total Stellar Mass for Different Metallicities and Regions

\begin{tabular}{lcc}
\hline \hline$Z$ & $M_{\text {tot }}\left(10^{6} M_{\odot}\right)$ & Regions \\
\hline 0.0012 & $9.5 \pm 4.2$ & $r_{h}$ \\
0.0012 & $17.7 \pm 7.9$ & $2 r_{h}$ \\
0.0007 & $13.3 \pm 5.3$ & $r_{h}$ \\
0.0007 & $15.4 \pm 6.2$ & $2 r_{h}$ \\
0.0004 & $20.6 \pm 8.4$ & $r_{h}$ \\
0.0004 & $26.8 \pm 10.1$ & $2 r_{h}$ \\
\hline
\end{tabular}

values obtained by previous studies, such as Kirby et al. (2020), who reported $M_{\mathrm{tot}}=(16.2 \pm 1.3) \times 10^{6} \quad M_{\odot}$, and McConnachie (2012), who obtained $M_{\text {tot }}=9.5 \times 10^{6} M_{\odot}$, but $M_{\text {tot }}=19.7 \times 10^{6} M_{\odot}$ in McConnachie et al. (2018). Furthermore, Weisz et al. (2014a) counted the total stellar mass formed within $0.19 r_{\frac{1}{2}}$ as $(12.8 \pm 1.5) \times 10^{6} M_{\odot}$. However, their shallow photometry made their SFH in this galaxy less secure. They found And VII to have an ancient SFH, while the presence of metal-rich, high- $[\alpha / \mathrm{Fe}]$ stars, which were found by Kirby et al. (2020) and Wojno et al. (2020), does not agree with an exclusively ancient SFH.

In order to follow back the SFH, it is necessary to take into account the variation of metallicity with age up to the quenching time. Since dSphs have been quenched early on, their chemical evolution did not significantly change at a later time (Skillman 2007). Metallicity of the ISM is a function of the look-back time of a galaxy and increases with decreasing $\alpha$ element abundance. However, And VII in all metallicity ranges exhibits a constant value of $[\alpha / \mathrm{Fe}] \simeq+0.3$ (Vargas et al. 2014 ) or shows a shallow negative slope in $[\alpha / \mathrm{Fe}]-[\mathrm{Fe} / \mathrm{H}]$ with a mean value of $[\alpha / \mathrm{Fe}] \simeq+0.18 \pm 0.03$ (Kirby et al. 2020; Wojno et al. 2020). By taking into account the $\alpha$-element abundance for the metallicity of $[\mathrm{Fe} / \mathrm{H}] \simeq-1.24 \pm 0.003 \mathrm{dex}$, we considered a metal-rich environment of $Z=0.0012$ for the young population. In contrast, for the old population $Z=0.0004$ was adopted, as stars with $[\mathrm{Fe} / \mathrm{H}] \in[-2.5,-1.5]$ have been found in And VII. Hence, Figures 15 and 14 show how the SFR changes for different assumptions of the metallicity: the SFRs for $t>8 \mathrm{Gyr}$ at $Z=0.0004$ and for $t<5$ Gyr at $Z=0.0012$ are larger than the corresponding SFR at $Z=0.0007$.

\subsection{Cumulative SFH and Quenching Time}

The cumulative SFH is built up of stellar mass over cosmological time:

$$
\text { Cumulative SFH } \pm \delta=\Sigma \frac{\mathrm{SFR} \pm \text { error }_{\mathrm{SFR}}}{M_{\text {cumulative }}} \times \Delta t,
$$

where $\Delta t$ specifies the length of each temporal bin and error SFR is that bin's uncertainty in SFR. The cumulative SFH of And VII, assuming the two metalicities $Z=0.0007$ and 0.0004 , is shown in Figure 16. From that, we can calculate the amount of stellar mass at any given time. Dwarf spheroidal galaxies hold very little gas, and the star formation in them has been extinguished many gigayears ago. The quenching time (hereafter $\tau_{90}$ ) for each of them is when $90 \%$ of the total stellar mass had been formed. By interpolating the cumulative SFH of And VII, $\tau_{90}$ is estimated about 5 Gyr ago $(\log t=9.7)$ with the present metal-rich condition $\left(\tau_{90}=4.97_{-2.30}^{+0.95}\right.$ Gyr ago with $Z=0.0007$ and $\tau_{90}=5.06_{-0.69}^{+1.09} \mathrm{Gyr}$ ago with $Z=0.0012$ ),

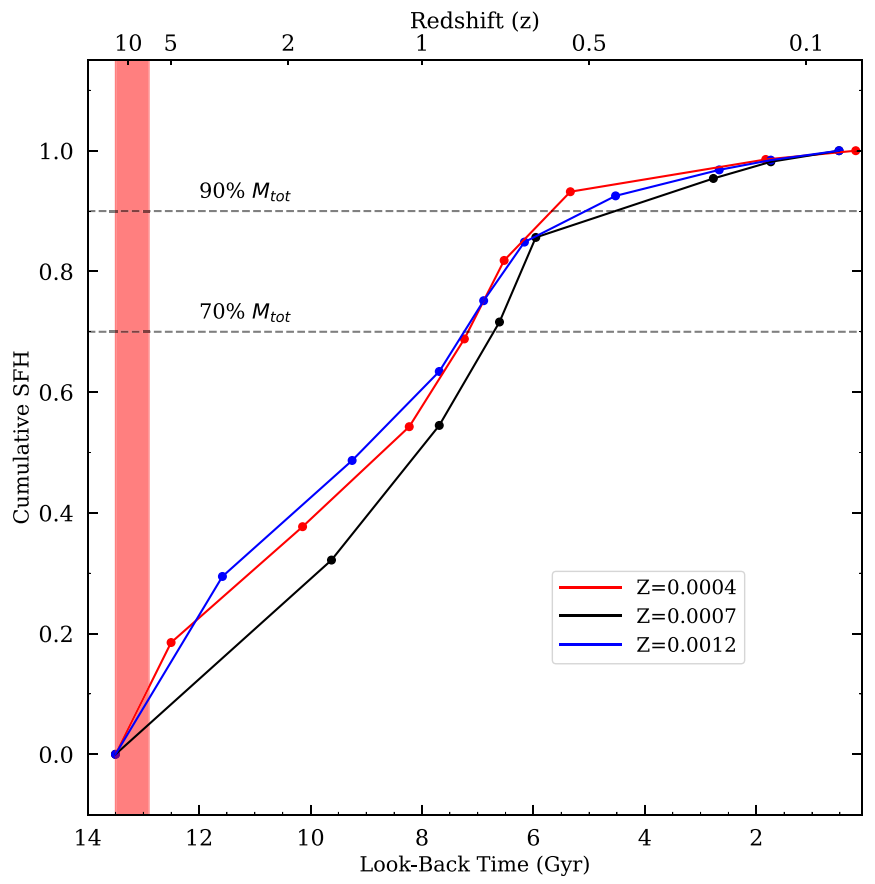

Figure 16. Cumulative SFH of And VII within $2 r_{\frac{1}{2}}$ for adopted metallicities of $Z=0.0007$ and 0.0004 . The red band indicates the epoch of reionization $(z \sim 6-14,12.9-13.5$ Gyr ago; Fan et al. 2006).

but if we assume that the galaxy initially formed iron-poor stars, the quenching time may have happened as early as $5.77_{-1.03}^{+0.73}$ Gyr ago $(\log t=9.76)$ with $Z=0.0004$.

Weisz et al. (2015) investigated the cumulative SFH of 38 dwarf galaxies in the Local Group and concluded that the mass of dwarfs plays an important role in the quenching time. Although they did not report the quenching time of And VII in their study, we can compare And VII to other M31 dSphs (And I, II, III, VI) with the same mass. The $\tau_{90}$ of these galaxies were found from 7.4 to $5.2 \mathrm{Gyr}$ ago $(\log (t / \mathrm{yr})=9.87-9.72)$ by Weisz et al. (2015), and the quenching time of And VII, with the largest mass, is later than most if not all of them. Our results confirm the observed relation that lower-mass dwarfs typically cease stellar mass assembly earlier than higher-mass galaxies (Weisz et al. 2015; Garrison-Kimmel et al. 2019). We note that these five galaxies are located within the M31 virial radius, where the quenching time is thus essentially independent of the current proximity to M31 (Weisz et al. 2015).

Although most M31 dSph satellites include old stellar populations with ages $\geqslant 10 \mathrm{Gyr}$, Harbeck et al. (2004) declared that And VII could possess an intermediate-age population owing to the evidence of carbon stars in this dwarf. Accordingly, they mentioned that star formation in And VII may have been active as recently as 3-5 Gyr ago. Also, they remarked based on the model of Mouhcine \& Lançon (2003) that there is no evidence of a notably younger intermediate-age $(<3 \mathrm{Gyr})$ stellar population in And VII. Moreover, Wetzel et al. (2015) applied the ELVIS suite to estimate the distribution of infall times for satellites with $M_{\text {star }}=10^{3-9} M_{\odot}$ at $z=0$. They realized that infall into the Milky Way/M31 halo typically happened 5-8 Gyr ago at $z=0.5-1$. Assuming that environmental quenching is related to infall time, And VII first fell into the M31 halo about 5-8 Gyr ago, leading to suppression of its star formation at $5 \mathrm{Gyr}$ ago. 
The red band in Figure 16 indicates the epoch of reionization ( $z \sim 6-14,12.9-13.5$ Gyr ago; Fan et al. 2006). The best fossil candidates at reionization are low-mass dwarfs $\left(M_{\text {star }} \lesssim 10^{6} M_{\odot}\right)$, where at least $70 \%$ of their stellar mass had been formed by the time of cosmic reionization. The dashed line in Figure 16 shows that $\tau_{70}$ (i.e., the epoch by which $70 \%$ of the total stellar mass had formed) was in place by $7 \mathrm{Gyr}$ ago at $Z=0.0007$ or $7.7 \mathrm{Gyr}$ ago at $Z=0.0004$. Therefore, it has not been presumed that quenching of And VII (with a large mass of $\sim 10^{7} M_{\odot}$ ) was affected by reionization. Most Local Group dwarfs did not form the bulk of their stellar mass during reionization (Weisz et al. 2014b), and probably environment has a stronger impact on the quenching of their star formation (Weisz et al. 2015).

Also, $\tau_{70}$ can define the duration and efficiency of star formation in dSphs. The relation between dark halo structure and SFH of several M31 dwarf galaxies was investigated based on comparisons between the density profile of dark halo $\left(\rho\left(b_{\text {halo }}\right)\right)$ and $\tau_{70}$ by Hayashi $\&$ Chiba (2015). Their aim was to figure out whether dark halo properties depend on the SFH of the stellar component. For this purpose, they adopted $\tau_{70}$ values from Weisz et al. (2014a), who reported $\tau_{70} \simeq 12.8 \mathrm{Gyr}$ for And VII, which caused this dwarf to be classified as a galaxy with a rapid SFH that holds a dense and concentrated dark halo. However, Weisz et al. (2014a) were not satisfied with the SFH result of And VII from Hubble Space Telescope WFPC2 data in their own research since the CMD of this galaxy was too shallow to show any age-sensitive features. Fortunately, in this study we could calculate the exact value of And VII's $\tau_{70}$ being between 7.0 and $7.7 \mathrm{Gyr}$; thus, the SFH of this galaxy has not happened as rapidly as Weisz et al. (2014a) predicted. Moreover, this value is more consistent with $\tau_{70}$ of other similar M31 dSphs (Weisz et al. 2014b). Also, $\rho\left(b_{\text {halo }}\right)$ of And VII is more similar to M31 dwarf galaxies with $\tau_{70}$ between 7 and 10 Gyr (Hayashi \& Chiba 2015).

\subsection{Galactocentric Radial Gradient of SFH}

The galactocentric radial gradient of SFH traces the compactness of star formation across cosmological time. The SFHs of And VII across different radius bins with an equal number of stars are displayed in Figure 17 at $Z=0.0007$. It is clear that there is not a distinct trend with radius, and star formation at any age has occurred at all distances. This result was also concluded by Vargas et al. (2014) based on the $\alpha$ element abundance gradient. Since And VII does not exhibit any evidence of an $[\alpha / \mathrm{Fe}]$ radial gradient, they concluded that the star formation timescales were not strongly dependent on the radial distance from the center of the galaxy. Figure 17 demonstrates that the peak of SFH occurred at all radii before $6 \mathrm{Gyr}$ ago and that its rate has been decreasing with increasing radial distance. The peak SFR declines by a factor of $\sim 15$ between the innermost and outermost regions $(\delta r \sim 1.25 \mathrm{kpc})$. The most recent star formation $(\log t / \mathrm{yr}<9.6)$ is more noticeable in the inner regions $(<0.5 \mathrm{kpc})$, suggesting that star formation may have been quenched outside-in.

\subsection{Minimum Birth Mass of Carbon Stars}

Since the majority of our candidate LPV stars do not have observational information to distinguish carbon stars from oxygen-rich (M-type) stars, we had to select a mass range for carbon stars based on theory (Padova model). However, the masses of carbon stars that were found by Harbeck et al. (2004)
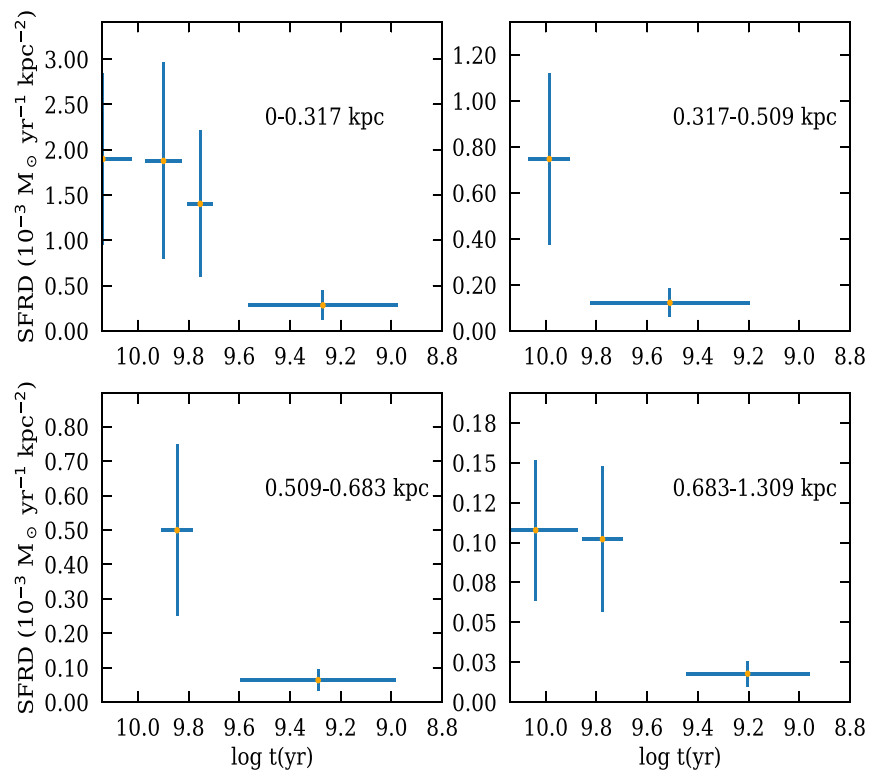

Figure 17. SFHs of And VII at constant $Z=0.0007$ in bins with equal numbers of LPV stars at galactocentric radii as indicated.

Table 6

Total Stellar Mass and Quenching Time for SFHs with Different Minimal Initial Mass for Carbon Stars

\begin{tabular}{lcc}
\hline \hline Birth Mass $M_{\odot}$ & $M_{\text {tot }}\left(10^{7} M_{\odot}\right)$ & $\tau_{90}(\mathrm{Gyr})$ \\
\hline 0.6 & $1.66 \pm 0.67$ & $5.2_{-0.4}^{+1.3}$ \\
0.7 & $1.71 \pm 0.70$ & $5.2_{-0.7}^{+1.0}$ \\
0.8 & $1.55 \pm 0.64$ & $5.1_{-0.7}^{+1.0}$ \\
0.9 & $1.50 \pm 0.62$ & $4.9_{-0.9}^{+1.1}$ \\
1.0 & $1.46 \pm 0.60$ & $4.8_{-1.1}^{+1.1}$ \\
1.1 & $1.44 \pm 0.59$ & $4.9_{-1.7}^{+1.3}$ \\
\hline
\end{tabular}

in And VII are obtained between 0.6 and 1.7 using our modeling. At a metallicity of $Z=0.004$ (typical of the Magellanic Clouds), the AGB stars with a minimum birth mass of $\sim 1.1 M_{\odot}$ were estimated to be a carbon star (e.g., Renzini \& Voli 1981; Groenewegen \& de Jong 1993; Dell'Agli et al. 2016). Also, Karakas et al. (2002) and Karakas (2014) predicted that at low metallicity the efficiency of dredge-up is higher and low-mass AGB stars can more easily turn into carbon stars.

Accordingly, at $Z=0.0007$ or lower, the mass range of carbon stars can be larger, and carbon stars might be produced from a minimum birth mass $<1.1 M_{\odot}$. Hence, we remeasured And VII's SFH based on carbon stars that arise from stars with a minimum of solar and subsolar initial mass (from 0.6 to $\left.1.1 M_{\odot}\right)$. The lower end of this range implies that the results are obtained using exclusively carbonaceous dust correction. In Figure 18, we show the SFH for the different mass range of carbon stars. Considering the subsolar carbon stars, the SFH would have experienced an exponential decrease, but without them, star formation in And VII suddenly decreased after the peak. In Table 6, the differences between total masses and quenching times are small. It appears that a different mass range for carbon stars does not substantively alter the inferred time of quenching but only has an impact on the manner in which it was quenching. 

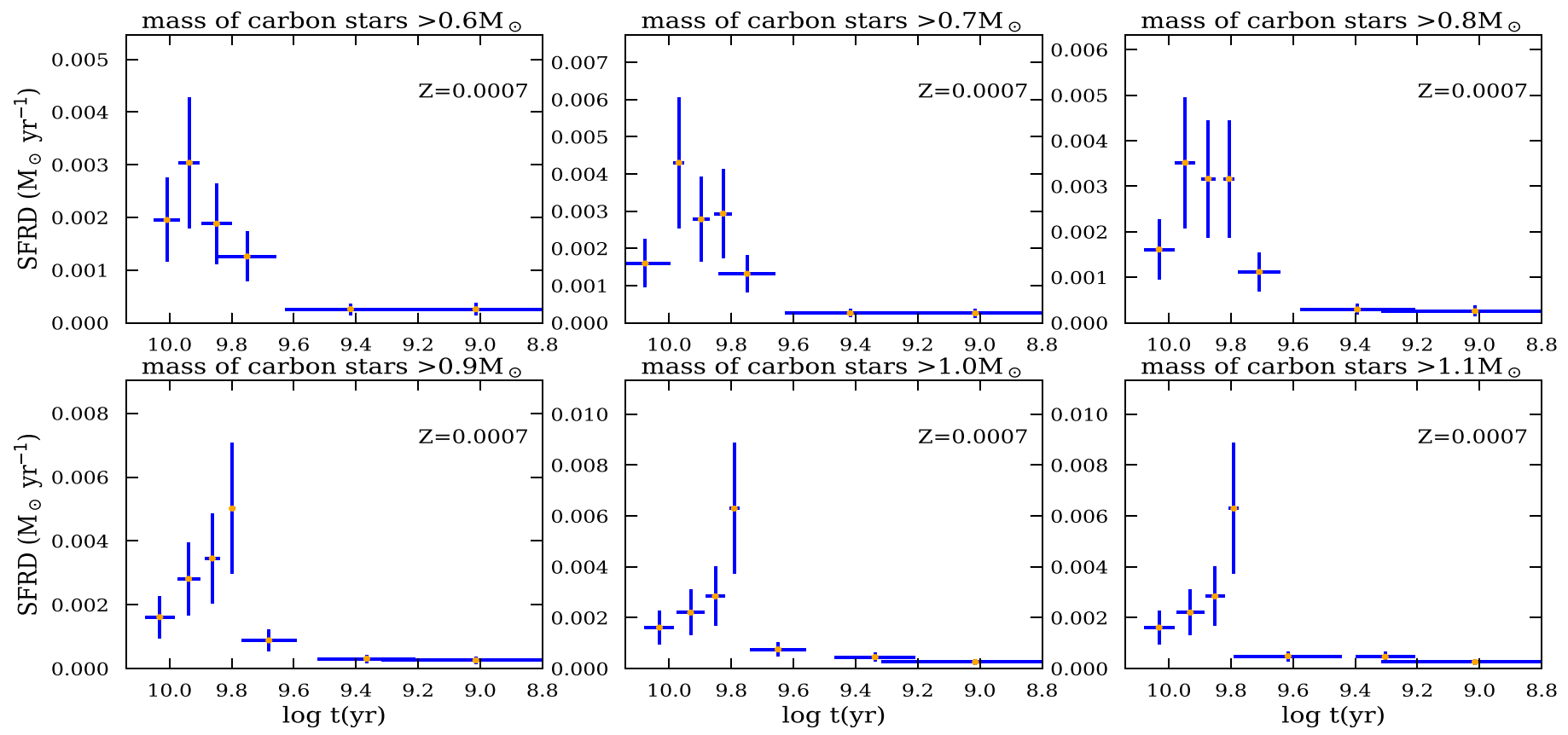

Figure 18. Remeasuring of And VII's SFH with different mass ranges for carbon stars. From the top left to the bottom right panels, the minimum of the mass range for C stars is decreasing (from 0.6 to $1.1 M_{\odot}$ ).

\section{Conclusions}

We have presented a photometric catalog in the $i$ and $V$ filters from new data obtained with INT/WFC of the And VII dwarf spheroidal galaxy. About 10,000 stars, including 55 candidate LPV stars, were detected within twice the half-light radius, determined as 3!.8 in this study. The AGB and RGB tips of the galaxy are identified. By combining the numbers and brightnesses of detected LPV stars with stellar isochrones, the SFH of And VII was reconstructed. The main epoch of star formation in And VII happened $\approx 6.2 \mathrm{Gyr}$ ago, and no evidence of star formation more recent than $400 \mathrm{Myr}$ ago can be found in this galaxy. About $70 \%$ of And VII's total stellar mass may have been assembled by $7 \mathrm{Gyr}$ ago, and $90 \%$ was in place by $5 \mathrm{Gyr}$ ago, when this dSph galaxy was quenched. The quenching of this galaxy occurred after reionization, and likely it was related to environmental impacts. Thus, And VII is dominated by an old (7-14 Gyr) stellar population. But also it includes metal-rich intermediate-age (5-7 Gyr) stars, which appears to be confirmed by the known presence of five carbon stars in this dwarf (Harbeck et al. 2004). Consequently,
And VII contains a young population of similar age to the stellar halo of M31. The total mass of stars that was formed during the galaxy's evolution is $(15.4 \pm 6.2) \times 10^{6} M_{\odot}$ within an elliptical radius of $2 r_{\frac{1}{2}} \simeq 1.5 \mathrm{kpc}$ from the center.

The observing time for this survey was primarily provided by the Iranian National Observatory (INO), complemented by UK-PATT allocation of time to programs I/2016B/09 and I/ 2017B/04 (PI: J. van Loon). We thank the INO and the School of Astronomy (IPM) for the financial support of this project. Authors are grateful to Peter Stetson for sharing his photometry routines. We thank James Bamber, Philip Short, Lucia SuárezAndrés, and Rosa Clavero for their help with the observations.

\section{Appendix}

The relations between birth mass and parametres such as $i$-band magnitude, age and pulsation duration for metallicites of $Z=0.0004$ and 0.0012 are presented in Figure 19 and Tables 7-9. 

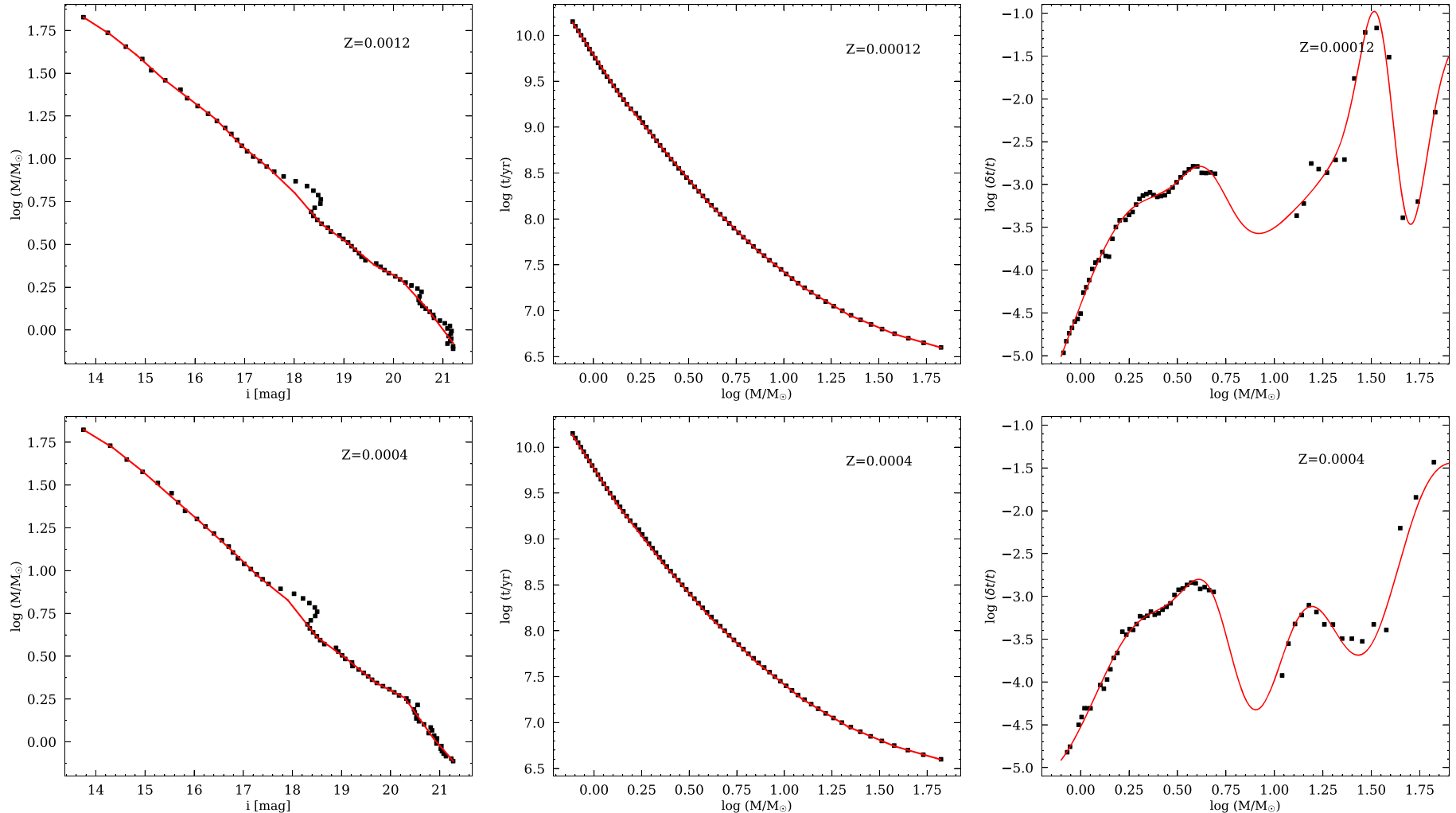

Figure 19. The relations between birth mass and parameters, such as $i$-band magnitude, age, and pulsation duration are shown from left to right panels, respectively, (at a distance modulus of $\mu=24.38 \mathrm{mag}$ for $Z=0.0012$ in the top row and for $Z=0.0004$ in the bottom row).

Table 7

Fitting Equations of the Relation between Birth Mass and $i$-band Magnitude, $\log M / M_{\odot}=a i+b$ for a Distance Modulus $\mu=24.38 \mathrm{mag}$

\begin{tabular}{|c|c|c|}
\hline$a$ & $\begin{array}{l}b \\
Z=0.0012\end{array}$ & Validity Range \\
\hline $\begin{array}{l}-0.186 \pm 0.063 \\
-0.227 \pm 0.064 \\
-0.260 \pm 0.062 \\
-0.221 \pm 0.055 \\
-0.223 \pm 0.047 \\
-0.296 \pm 0.042 \\
-0.239 \pm 0.042 \\
-0.277 \pm 0.094 \\
-0.338 \pm 0.091 \\
-0.210 \pm 0.037 \\
-0.240 \pm 0.037 \\
-0.155 \pm 0.039 \\
-0.322 \pm 0.034 \\
-0.382 \pm 0.027\end{array}$ & $\begin{array}{r}4.383 \pm 0.922 \\
4.968 \pm 0.967 \\
5.461 \pm 0.972 \\
4.863 \pm 0.879 \\
4.889 \pm 0.786 \\
6.085 \pm 0.714 \\
5.124 \pm 0.737 \\
5.799 \pm 1.724 \\
6.892 \pm 1.699 \\
4.520 \pm 0.710 \\
5.081 \pm 0.727 \\
3.418 \pm 0.803 \\
-6.522 \pm 0.698 \\
-8.165 \pm 0.572\end{array}$ & $\begin{array}{c}i \leqslant 14.285 \\
14.285<i \leqslant 14.817 \\
14.817<i \leqslant 15.349 \\
15.349<i \leqslant 15.882 \\
15.822<i \leqslant 16.414 \\
16.414<i \leqslant 16.946 \\
16.946<i \leqslant 17.478 \\
17.478<i \leqslant 18.010 \\
18.010<i \leqslant 18.542 \\
18.542<i \leqslant 19.075 \\
19.075<i \leqslant 19.607 \\
19.607<i \leqslant 20.139 \\
20.139<i \leqslant 20.671 \\
i>20.671\end{array}$ \\
\hline$a$ & $\begin{array}{l}b \\
Z=0.0004\end{array}$ & Validity Range \\
\hline $\begin{array}{l}-0.176 \pm 0.052 \\
-0.228 \pm 0.049 \\
-0.253 \pm 0.050 \\
-0.251 \pm 0.047 \\
-0.259 \pm 0.035 \\
-0.279 \pm 0.033 \\
-0.231 \pm 0.120 \\
-0.363 \pm 0.118 \\
-0.211 \pm 0.029 \\
-0.235 \pm 0.027 \\
-0.150 \pm 0.027 \\
-0.403 \pm 0.022 \\
-0.220 \pm 0.029\end{array}$ & $\begin{array}{l}4.242 \pm 0.754 \\
4.983 \pm 0.741 \\
5.356 \pm 0.790 \\
5.325 \pm 0.762 \\
5.465 \pm 0.600 \\
5.792 \pm 0.589 \\
4.956 \pm 2.205 \\
7.334 \pm 2.186 \\
4.513 \pm 0.563 \\
4.971 \pm 0.545 \\
3.309 \pm 0.546 \\
8.425 \pm 0.462 \\
-4.795 \pm 0.596\end{array}$ & $\begin{array}{c}i \leqslant 14.342 \\
14.342<i \leqslant 14.936 \\
14.936<i \leqslant 15.530 \\
15.530<i \leqslant 16.125 \\
16.125<i \leqslant 16.719 \\
16.719<i \leqslant 17.313 \\
17.313<i \leqslant 17.907 \\
17.907<i \leqslant 18.501 \\
18.501<i \leqslant 19.095 \\
19.095<i \leqslant 19.689 \\
19.689<i \leqslant 20.284 \\
20.284<i \leqslant 20.878 \\
i>20.878\end{array}$ \\
\hline
\end{tabular}


Table 8

Relation between Age and Birth Mass, $\log t=a \log M+b$

\begin{tabular}{lcc}
\hline \hline$a$ & $b$ & Validity Range \\
& $Z=0.0012$ & \\
\hline$-3.189 \pm 0.024$ & $9.788 \pm 0.006$ & $\log M \leqslant 0.133$ \\
$-2.594 \pm 0.022$ & $9.709 \pm 0.011$ & $0.133<\log M \leqslant 0.375$ \\
$-2.441 \pm 0.023$ & $9.652 \pm 0.017$ & $0.375<\log M \leqslant 0.617$ \\
$-2.002 \pm 0.025$ & $9.382 \pm 0.025$ & $0.617<\log M \leqslant 0.859$ \\
$-1.680 \pm 0.028$ & $9.105 \pm 0.034$ & $0.859<\log M \leqslant 1.101$ \\
$-1.248 \pm 0.032$ & $8.629 \pm 0.047$ & $1.101<\log M \leqslant 1.343$ \\
$-0.867 \pm 0.037$ & $8.118 \pm 0.064$ & $1.343<\log M \leqslant 1.585$ \\
$-0.601 \pm 0.045$ & $7.696 \pm 0.088$ & $\log M>1.585$ \\
\hline$a$ & & \\
& & $\operatorname{Validity~Range}$ \\
& & \\
\hline$-3.201 \pm 0.022$ & $9.776 \pm 0.005$ & $\log M \leqslant 0.129$ \\
$-2.616 \pm 0.020$ & $9.700 \pm 0.010$ & $0.129<\log M \leqslant 0.371$ \\
$-2.404 \pm 0.021$ & $9.622 \pm 0.016$ & $0.371<\log M \leqslant 0.613$ \\
$-2.006 \pm 0.023$ & $9.377 \pm 0.023$ & $0.613<\log M \leqslant 0.855$ \\
$-1.685 \pm 0.026$ & $9.103 \pm 0.031$ & $0.855<\log M \leqslant 1.097$ \\
$-1.249 \pm 0.029$ & $8.625 \pm 0.043$ & $1.097<\log M \leqslant 1.339$ \\
$-0.869 \pm 0.035$ & $8.116 \pm 0.059$ & $1.339<\log M \leqslant 1.581$ \\
$-0.595 \pm 0.042$ & $7.683 \pm 0.082$ & $\log M>1.581$ \\
\hline
\end{tabular}

Table 9

Fits to the Relation between Relative Pulsation Duration $(\delta t / t$, where $t$ is the Age and $\delta t$ is the Pulsation Duration) and Birth Mass, $\log (\delta t / t)=\Sigma_{i=1}^{5} a_{i} \exp \left[-\left(\log M\left[M_{\odot}\right]-b_{i}\right)^{2} / c_{i}^{2}\right]$

\begin{tabular}{|c|c|c|c|}
\hline $\bar{i}$ & $a$ & $b$ & $c$ \\
\hline \multicolumn{4}{|c|}{$Z=0.0012$} \\
\hline 1 & 0.7591 & 0.6472 & 0.1710 \\
\hline 2 & 2.6567 & 0.2388 & 0.4794 \\
\hline 3 & -27.203 & 1.61884 & 0.14442 \\
\hline 4 & -6.8127 & -0.4083 & 1.81376 \\
\hline 5 & 27.3586 & 1.60982 & 0.14418 \\
\hline \multicolumn{4}{|c|}{$Z=0.0004$} \\
\hline 1 & -32.369 & 6.8349 & 5.4629 \\
\hline 2 & 0.4006 & 0.2669 & 0.0976 \\
\hline 3 & 35.049 & 1.3629 & 0.8449 \\
\hline 4 & -1.5269 & 1.5129 & 0.1107 \\
\hline 5 & -27.069 & 1.2729 & 0.6624 \\
\hline
\end{tabular}

\section{ORCID iDs}

Jacco Th. van Loon (ib https://orcid.org/0000-0002-1272-3017 Mojtaba Raouf (iD https://orcid.org/0000-00021496-3591

\section{References}

Battinelli, P., \& Demers, S. 2006, A\&A, 447, 473

Bladh, S., Liljegren, S., Hoefner, S., Aringer, B., \& Marigo, P. 2019, A\&A, 626, A100

Boyer, M. L., McQuinn, K. B. W., Barmby, P., et al. 2015, ApJS, 216, 10 Bressan, A., Marigo, P., Girardi, L., et al. 2012, MNRAS, 427, 127

Cole, A. A., Skillman, E. D., Tolstoy, E., et al. 2007, ApJL, 659, L17

Dell'Agli, F., Di Criscienzo, M., Boyer, M. L., \& García-Hernández, D. A. 2016, MNRAS, 460, 4230

Dell'Agli, F., Di Criscienzo, M., Ventura, P., et al. 2018, MNRAS, 479, 5035 Dolphin, A. 1997, NewA, 2, 397

Draine, B. T., \& Li, A. 2007, ApJ, 657, 810

Fan, X., Carilli, C. L., \& Keating, B. 2006, ARA\&A, 44, 415

Gaia Collaboration, Brown, A. G. A., Vallenari, A., et al. 2018, A\&A, 616, A1
Garrison-Kimmel, S., Wetzel, A., Hopkins, P. F., et al. 2019, MNRAS, 489, 4574

Gilbert, K. M., Wojno, J., Kirby, E. N., et al. 2020, AJ, 160, 41

Girardi, L., Groenewegen, M. A. T., Hatziminaoglou, E., \& da Costa, L. 2005, A\&A, 436, 895

Grcevich, J., \& Putman, M. E. 2009, ApJ, 696, 385

Grebel, E. K., \& Guhathakurta, P. 1999, ApJL, 511, L101

Groenewegen, M. A. T., \& de Jong, T. 1993, A\&A, 267, 410

Hamedani Golshan, R., Javadi, A., van Loon, J. T., Khosroshahi, H., \& Saremi, E. 2017, MNRAS, 466, 1764

Hamren, K., Beaton, R. L., Guhathakurta, P., et al. 2016, ApJ, 828, 15

Harbeck, D., Grebel, E., Gallagher, J., Koch, A., \& Zucker, D. 2004, AAS Meeting Abstracts, 205, 93.01

Hashemi, S. A., Javadi, A., \& van Loon, J. T. 2019, MNRAS, 483, 4751

Hayashi, K., \& Chiba, M. 2015, IAUGA, 29, 2254520

Höfner, S., \& Olofsson, H. 2018, A\&ARv, 26, 1

Iben, I. J., \& Renzini, A. 1983, ARA\&A, 21, 271

Javadi, A., van Loon, J. T., Khosroshahi, H. G., et al. 2017, MNRAS, 464,2103

Javadi, A., van Loon, J. T., \& Mirtorabi, M. T. 2011a, MNRAS, 411, 263

Javadi, A., van Loon, J. T., \& Mirtorabi, M. T. 2011b, MNRAS, 414, 3394

Jordi, K., Grebel, E. K., \& Ammon, K. 2006, A\&A, 460, 339

Kalirai, J. S., Beaton, R. L., Geha, M. C., et al. 2010, ApJ, 711, 671

Karachentsev, I., Aparicio, A., \& Makarova, L. 1999, A\&A, 352, 363

Karachentsev, I. D., Sharina, M. E., Dolphin, A. E., \& Grebel, E. K. 2003, A\&A, 408, 111

Karakas, A. I. 2014, MNRAS, 445, 347

Karakas, A. I., Lattanzio, J. C., \& Pols, O. R. 2002, PASA, 19, 515

Kirby, E. N., Gilbert, K. M., Escala, I., et al. 2020, AJ, 159, 46

Kroupa, P. 2001, MNRAS, 322, 231

Lee, M. G., Freedman, W. L., \& Madore, B. F. 1993, ApJ, 417, 553

Levesque, E. M. 2010, in ASP Conf. Ser. 425, The Physical Properties of Red Supergiants, ed. C. Leitherer et al. (San Francisco, CA: ASP), 103

Lupton, R. 2005, Transformations between SDSS magnitudes and UBVRcIc, http://classic.sdss.org/dr6/algorithms/sdssUBVRITransform. html\#Lupton2005

Levesque, E. M., Massey, P., Olsen, K. A. G., et al. 2005, AAS Meeting Abstracts, 207, 182.13

Marigo, P., Girardi, L., Bressan, A., et al. 2008, A\&A, 482, 883

Marigo, P., Girardi, L., Bressan, A., et al. 2017, ApJ, 835, 77

McConnachie, A. W. 2012, AJ, 144, 4

McConnachie, A. W., Ibata, R., Martin, N., et al. 2018, ApJ, 868, 55

McConnachie, A. W., \& Irwin, M. J. 2006, MNRAS, 365, 1263

McConnachie, A. W., Irwin, M. J., Ferguson, A. M. N., et al. 2004, MNRAS, 350,243

McConnachie, A. W., Irwin, M. J., Ferguson, A. M. N., et al. 2005, MNRAS, 356, 979

McDonald, I., van Loon, J. T., Sloan, G. C., et al. 2011, MNRAS, 417, 20

McDonald, I., White, J. R., Zijlstra, A. A., et al. 2012, MNRAS, 427, 2647

McDonald, I., \& Zijlstra, A. A. 2016, ApJL, 823, L38

Mouhcine, M., \& Lançon, A. 2003, MNRAS, 338, 572

Navabi, M., Noori, M., Saremi, E., et al. 2020, in Proc. Conf. Stars and their Variability Observed from Space, ed. C. Neiner et al. (Vienna: Univ. Vienna), 383

Ohnaka, K., Weigelt, G., \& Hofmann, K.-H. 2017, A\&A, 597, A20

Pastorelli, G., Marigo, P., Girardi, L., et al. 2019, MNRAS, 485, 5666

Renzini, A., \& Voli, M. 1981, A\&A, 500, 221

Rezaeikh, S., Javadi, A., Khosroshahi, H., \& van Loon, J. T. 2014, MNRAS, 445, 2214

Samus', N. N., Durlevich, O. V., Zharova, A. V., et al. 2006, AstL, 32, 263

Saremi, E., Javadi, A., Th. van Loon, J., et al. 2020, ApJ, 894, 135

Saremi, E., Javadi, A., van Loon, J., Khosroshahi, H., \& Torki, M. 2019a, in IAU Symp. 339, Southern Horizons in Time-Domain Astronomy (Cambridge: Cambridge Univ. Press), 336

Saremi, E., Javadi, A., van Loon, J. T., et al. 2017, JPhCS, 869, 012068

Saremi, E., Javadi, A., van Loon, J. T., et al. 2019b, in IAU Symp. 344, Dwarf Galaxies: From the Deep Universe to the Present (Cambridge: Cambridge Univ. Press), 125

Skillman, E. D. 2007, in IAU Symp. 235, Galaxy Evolution across the Hubble Time, ed. F. Combes \& J. Palouš (Cambridge: Cambridge Univ. Press), 57

Skillman, E. D., Monelli, M., Weisz, D. R., et al. 2017, ApJ, 837, 102

Stetson, P. B. 1987, PASP, 99, 191

Stetson, P. B. 1990, PASP, 102, 932

Stetson, P. B. 1996, PASP, 108, 851

Tej, A., Lançon, A., \& Scholz, M. 2003, A\&A, 401, 347

Tollerud, E. J., Beaton, R. L., Geha, M. C., et al. 2012, ApJ, 752, 45 
van Loon, J. T., van Leeuwen, F., Smalley, B., et al. 2007, MNRAS, 382, 1353

Vargas, L. C., Geha, M. C., \& Tollerud, E. J. 2014, ApJ, 790, 73

Weisz, D. R., Dolphin, A. E., Skillman, E. D., et al. 2014a, ApJ, 789, 147

Weisz, D. R., Dolphin, A. E., Skillman, E. D., et al. 2014b, ApJ, 789, 148

Weisz, D. R., Dolphin, A. E., Skillman, E. D., et al. 2015, ApJ, 804, 136

Welch, D. L., \& Stetson, P. B. 1993, AJ, 105, 1813
Wetzel, A. R., Deason, A. J., \& Garrison-Kimmel, S. 2015, ApJ, 807, 49

Wojno, J., Gilbert, K. M., Kirby, E. N., et al. 2020, ApJ, 895, 78

Wood, P. R., Whiteoak, J. B., Hughes, S. M. G., et al. 1992, ApJ, 397, 552

Yuan, W., Macri, L. M., Javadi, A., Lin, Z., \& Huang, J. Z. 2018, AJ, 156, 112

Zhao-Geisler, R., Quirrenbach, A., Köhler, R., \& Lopez, B. 2012, A\&A, 545, A56 